\title{
Joint statistics of amplitudes and phases in wave turbulence
}

\author{
Yeontaek Choi ${ }^{\mathrm{a}}$, Yuri V. Lvov ${ }^{\mathrm{b}, *}$, Sergey Nazarenko ${ }^{\mathrm{a}}$ \\ a Mathematics Institute, The University of Warwick, Coventry CV4 7AL, UK \\ ${ }^{\mathrm{b}}$ Department of Mathematical Sciences, Rensselaer Polytechnic Institute, Troy, NY 12180-3590, USA
}

Received 2 June 2004; received in revised form 7 September 2004; accepted 23 November 2004

Communicated by U. Frisch

\begin{abstract}
Random Phase Approximation (RPA) provides a very convenient tool to study the ensembles of weakly interacting waves, commonly called wave turbulence. In its traditional formulation, RPA assumes that phases of interacting waves are random quantities but it usually ignores randomness of their amplitudes. Recently, RPA was generalised in a way that takes into account the amplitude randomness and it was applied to study of the higher momenta and probability densities of wave amplitudes. However, to have a meaningful description of wave turbulence, the RPA properties assumed for the initial fields must be proven to survive over the nonlinear evolution time, and such a proof is the main goal of the present paper. We derive an evolution equation for the full probability density function which contains the complete information about the joint statistics of all wave amplitudes and phases. We show that, for any initial statistics of the amplitudes, the phase factors remain statistically independent uniformly distributed variables. If in addition the initial amplitudes are also independent variables (but with arbitrary distributions) they will remain independent when considered in small sets which are much less than the total number of modes. However, if the size of a set is of order of the total number of modes then the joint probability density for this set is not factorisable into the product of one-mode probabilities. In the other words, the modes in such a set are involved in a "collective" (correlated) motion. We also study new type of correlators describing the phase statistics.
\end{abstract}

(C) 2004 Elsevier B.V. All rights reserved.

\section{Introduction}

Wave turbulence (WT) is a common name for the fields of dispersive waves which are engaged in stochastic weakly nonlinear interactions over a wide range of scales. Plentiful examples of WT are found in oceans, atmospheres, plasmas and Bose-Einstein condensates [1-7]. Roughly, there have been three major approaches to derive the WT theory, one based on a diagrammatic approach $[8,10,9]$, the second based on cumulant expansions $[2,4,11,7]$ and the third one, the Random Phase Approximation (RPA) [1,3,5].

\footnotetext{
* Corresponding author.

E-mail address: 1vov@rpi.edu (Y.V. Lvov).
} 
The diagrammatic approach was developed in a field theoretical spirit based on the Wyld's technique [8]. This method introduces an artificial Gaussian forcing for which a zero limit is taken at the end of the derivation. It is usually said that the statistical properties of this force (Gaussianity) do not affect the statistical properties of the resulting WT state which will be determined by the nonlinear properties only. However, such independence of the WT state on the statistics of the "seed" forcing is not obvious because the limit of small nonlinearity is taken before the limit of small force, i.e., the force remains much greater than the nonlinearity. In particular, when the nonlinearity parameter is strictly zero, the Wyld technique gives a Gaussian steady state which is clearly an artefact of this method because for linear systems statistics of the wave amplitudes remain the same as in the initial condition and, therefore, can be arbitrary. The question if any nonlinearity, no matter how small, can break this dependence of the steady state on the initial conditions still has not been answered in the literature. Thus, the diagrammatic approach, although a very efficient way to build the perturbation expansion, needs to be expanded to include non-Gaussian "seed" force in order to see to what extent the results are not sensitive to the force statistics. However, some elements of the Wyld technique will be used in the present paper, not as a complete description but rather as an auxiliary aid in writing out complicated terms.

The cumulant expansion approach differs from the other methods by working directly with the continuous Fourier transforms corresponding to the infinite coordinate space without introducing a finite box as an intermediate step. The main idea here is that, although the Fourier transform is ill-defined for the wave fields corresponding to homogeneous turbulence, it is well defined for the cumulants provided that the correlations decay rapidly enough in the coordinate space. The cumulant method is very elegant for describing the spectra and the multiple-point moments the points in which are not "fused" (i.e., all different). However, some important statistical quantities involve fused moments and they are hard (if at all possible) to define without introducing a finite box as an intermediate step. For example, one of such objects, $\left\langle|a|^{4}\right\rangle$, is important because it describes intensity of fluctuations of the $k$-space distribution of energy $|a|^{2}$, namely $\delta E=\sqrt{\left\langle|a|^{4}\right\rangle-\left\langle|a|^{2}\right\rangle^{2}}$ (see [19]). Furthermore, non-decaying in the $x$-space correlations tend to naturally develop over the nonlinear time [19] and it is not clear what wave fields these correlations correspond to within the cumulant approach.

RPA approach has been by far most popular technique due to its clear intuitive content. However, this approach has occasionally been downgraded to just a convenient way of interpreting the results of a more rigorous technique based on the cumulant expansions. It happened because RPA, being widely used by physicists, had not been formulated rigorously. In particular, it is typically assumed that the phases evolve much faster than amplitudes in the system of nonlinear dispersive waves and, therefore, the averaging may be made over the phases only "forgetting" that the amplitudes are statistical quantities too (see, e.g., [1]). This statement become less obvious if one takes into account that we are talking not about the linear phases $\omega t$ but about the phases of the Fourier modes in the interaction representation. Thus, it has to be the nonlinear frequency correction that helps randomising the phases [17]. On the other hand, for three-wave systems (considered in this paper) the period associated with the nonlinear frequency correction is of the same $\epsilon^{2}$ order in small nonlinearity $\epsilon$ as the nonlinear evolution time and, therefore, phase randomisation cannot occur faster that the nonlinear evolution of the amplitudes. One could hope that the situation is better for four-wave systems (not considered here) because the nonlinear frequency correction is still $\sim \epsilon^{2}$ but the nonlinear evolution appears only in the $\epsilon^{4}$ order. However, in order to make the asymptotic analysis consistent, such $\epsilon^{2}$ correction has to be removed from the interaction-representation amplitudes and the remaining phase and amplitude evolutions are, again, at the same time scale (now $1 / \epsilon^{4}$ ). This picture is confirmed by the numerical simulations of the four-wave systems $[18,20]$ which indicate that the nonlinear phase evolves at the same time scale as the amplitude. Thus, to proceed theoretically one has to start with phases which are already random (or almost random) and hope that this randomness is preserved over the nonlinear evolution time. In most of the previous literature such preservation was assumed but not proven. The goal of this paper will be to study the extent to which such an assumption is valid.

Another goal of this paper is to make RPA formulation more consistent by taking into account that both phases and the amplitudes are random variables. Indeed, even if one starts with a wavefield which has random phases but deterministic amplitudes, as it is typically done in numerical simulations, the amplitudes will get randomised because 
the nonlinear term producing their evolution contains (random) phase factors. Preliminary steps were recently done in $[19,20]$ where we assumed that all the phases and the amplitudes in the initial wavefield are random variables independent of each other and that the phase factors are uniformly distributed on the unit circle on the complex plane. We kept the same acronym RPA but re-interpreted it as "Random Phases and Amplitudes", reflecting the fact that, first, the amplitudes are also random and, second, that it is not an "approximation" but rather an assumed property of the initial field. Such a generalised RPA was used in [19] to study the evolution of the higher moments of the Fourier amplitudes and in [20] to study their "one-mode" PDF. In fact, this form of RPA is more general than the cumulant approach because it can handle fields with long correlation lengths which appear to be important for intermittency [20].

Of course, for such an analysis to be trustworthy one should prove that the RPA properties hold over the nonlinear time and not just for the initial fields. Such mathematical validation of the RPA method will be in the focus of the present paper. To do this we will have to study the full joint PDF which involves the complete statistical information about the system, including the multi-mode correlations of both the amplitudes and the phase factors. We will derive an evolution equation for such PDF and we will show that it is identical to the equation obtained for the excitations in anharmonic crystals originally obtained by Peierls [15] and later reproduced by Brout and Prigogine [16] and Zaslavskii and Sagdeev [17]. All these works were restricted to considering a quite narrow class of interaction Hamiltonians arising from a potential energy, i.e., depending on the coordinates but not momenta. These class does not include a large number of interesting WT systems, e.g., the capillary, internal, Rossby and Alfven waves. It is remarkable, therefore, that the Peierls equation turns out to be universal for the general class of three-wave systems, as it is shown in the present paper. Further, we use this equation to validate an "essential" RPA formulation, i.e., approximate RPA which holds only up to a certain order in nonlinearity and discreteness, but which is sufficient for the WT closure. This validation gives RPA technique a status of a rigorous approach which, due to the simplicity of its premises, is a winning tool for the future theory of non-Gaussianity of WT, its intermittency and interactions with coherent structures.

In addition to the mathematical validation of RPA, we will also develop WT further by considering new statistically important quantities. For a long time, describing and predicting the energy spectra were the only concern in WT theory. Recently, we presented a description of the higher order statistics of the one-point Fourier correlators in terms of their moments and PDFs. They describe the $k$-space "noise", i.e., the fluctuations of the mode energy about its mean value given by the energy spectrum. We also showed PDFs have a long algebraic tail which indicates presence of intermittency in WT fields. The present paper deals with phases, and we will therefore introduce and study some new correlators which will allow to describe the phase statistics directly. Such a description will compliment the mathematical validation of the RPA because it yields to a physical answer on how initially correlated phases can get de-correlated in the first place.

\section{Fields with Random Phases and Amplitudes}

Let us consider a wavefield $a(\mathbf{x}, t)$ in a periodic cube of with side $L$ and let the Fourier transform of this field be $a_{l}(t)$, where index $l \in \mathcal{Z}^{d}$ marks the mode with wavenumber $k_{l}=2 \pi l / L$ on the grid in the $d$-dimensional Fourier space. For simplicity let us assume that there is a maximum wavenumber $k_{\max }$ (fixed, e.g., by dissipation) so that no modes with wavenumbers greater than this maximum value can be excited. In this case, the total number of modes is $N=\left(k_{\max } / \pi L\right)^{d}$. Correspondingly, index $l$ will only take values in a finite box, $l \in \mathcal{B}_{N} \subset \mathcal{Z}^{d}$ which is centred at 0 and all sides of which are equal to $k_{\max } / \pi L=N^{1 / 3}$. To consider homogeneous turbulence, the large box limit $N \rightarrow \infty$ will have to be taken. ${ }^{1}$

\footnotetext{
${ }^{1}$ It is easy to extend the analysis to the infinite Fourier space, $k_{\max }=\infty$. In this case, the full joint PDF would still have to be defined as a $N \rightarrow \infty$ limit of an $N$-mode PDF, but this limit would have to be taken in such a way that both $k_{\max }$ and the density of the Fourier modes tend to infinity simultaneously.
} 
Let us write the complex $a_{l}$ as $a_{l}=A_{l} \psi_{l}$, where $A_{l}$ is a real positive amplitude and $\psi_{l}$ is a phase factor which takes values on $\mathcal{S}^{1}$, a unit circle centred at zero in the complex plane. Let us define the $N$-mode joint PDF $\mathcal{P}^{(N)}$ as the probability for the wave intensities $A_{l}^{2}$ to be in the range $\left(s_{l}, s_{l}+\mathrm{d} s_{l}\right)$ and for the phase factors $\psi_{l}$ to be on the unit-circle segment between $\xi_{l}$ and $\xi_{l}+\mathrm{d} \xi_{l}$ for all $l \in \mathcal{B}_{N}$. In terms of this PDF, taking the averages will involve integration over all the real positive $s_{l}$ 's and along all the complex unit circles of all $\xi_{l}$ 's,

$$
\left\langle f\left\{A^{2}, \psi\right\}\right\rangle=\left(\prod_{l \in \mathcal{B}_{N}} \int_{\mathcal{R}^{+}} \mathrm{d} s_{l} \oint_{\mathcal{S}^{1}}\left|\mathrm{~d} \xi_{l}\right|\right) \mathcal{P}^{(N)}\{s, \xi\} f\{s, \xi\},
$$

where notation $f\left\{A^{2}, \psi\right\}$ means that $f$ depends on all $A_{l}^{2}$ 's and all $\psi_{l}$ 's in the set $\left\{A_{l}^{2}, \psi_{l} ; l \in \mathcal{B}_{N}\right\}$ (similarly, $\{s, \xi\}$ means $\left\{s_{l}, \psi_{l} ; l \in \mathcal{B}_{N}\right\}$, etc). The full PDF that contains the complete statistical information about the wavefield $a(\mathbf{x}, t)$ in the infinite $x$-space can be understood as a large-box limit

$$
\mathcal{P}\left\{s_{k}, \xi_{k}\right\}=\lim _{N \rightarrow \infty} \mathcal{P}^{(N)}\{s, \xi\},
$$

i.e., it is a functional acting on the continuous functions of the wavenumber, $s_{k}$ and $\xi_{k}$. In the large box limit there is a path-integral version of (1),

$$
\left\langle f\left\{A^{2}, \psi\right\}\right\rangle=\int \mathcal{D} s \oint|\mathcal{D} \xi| \mathcal{P}\{s, \xi\} f\{s, \xi\} .
$$

The full PDF defined above involves all $N$ modes (for either finite $N$ or in the $N \rightarrow \infty$ limit). By integrating out all the arguments except for chosen few, one can have reduced statistical distributions. For example, by integrating over all the angles and over all but $M$ amplitudes, we have an " $M$-mode" amplitude PDF,

$$
\mathcal{P}_{j_{1}, j_{2}, \ldots, j_{M}}=\left(\prod_{l \neq j_{1}, j_{2}, \ldots, j_{M}} \int_{\mathcal{R}^{+}} \mathrm{d} s_{l} \prod_{m \mathcal{B}_{N}} \oint_{\mathcal{S}^{1}}\left|\mathrm{~d} \xi_{m}\right|\right) \mathcal{P}^{(N)}\{s, \xi\},
$$

which depends only on the $M$ amplitudes marked by labels $j_{1}, j_{2}, \ldots, j_{M} \in \mathcal{B}_{N}$.

\subsection{Definition of an ideal RPA field}

Following the approach of $[19,20]$, we now define a "Random Phase and Amplitude" field. We say that the field $a$ is of RPA type if it possesses the following statistical properties:

1. All amplitudes $A_{l}$ and their phase factors $\psi_{l}$ are independent random variables, i.e., their joint PDF is equal to the product of the one-mode PDFs corresponding to each individual amplitude and phase,

$$
\mathcal{P}^{(N)}\{s, \xi\}=\prod_{l \in \mathcal{B}_{N}} P_{l}^{(a)}\left(s_{l}\right) P_{l}^{(\psi)}\left(\xi_{l}\right)
$$

2. The phase factors $\psi_{l}$ are uniformly distributed on the unit circle in the complex plane, i.e., for any mode $l$

$$
P_{l}^{(\psi)}\left(\xi_{l}\right)=\frac{1}{2} \pi
$$

Note that RPA does not fix any shape of the amplitude PDFs and, therefore, can deal with strongly non-Gaussian wavefields. Such study of non-Gaussianity and intermittency of WT was presented in $[19,20]$ and will not be repeated here. However, we will study some new objects describing statistics of the phase. 
In $[19,20]$ RPA was assumed to hold over the nonlinear time. The main goal of this paper is to find out whether it is true that the RPA property survives over the nonlinear time and to what extent. We will see that RPA fails to hold in its pure form as formulated above but it survives in the leading order so that the WT closure built using the RPA is valid. We will also see that independence of the phase factors is quite straightforward, whereas the amplitude independence is subtle. Namely, $M$ amplitudes are independent only up to a $\mathrm{O}(M / N)$ correction. Based on this knowledge, and leaving justification for later on in this paper, we thus reformulate RPA in a weaker form which holds over the nonlinear time and which involves $M$-mode PDFs with $M \ll N$ rather than the full $N$-mode PDF.

\subsection{Definition of an essentially RPA field}

We will say that the field $a$ is of an "essentially RPA" type if:

1. The phase factors are statistically independent and uniformly distributed variables up to $\mathrm{O}\left(\epsilon^{2}\right)$ corrections, i.e.,

$$
\mathcal{P}^{(N)}\{s, \xi\}=\frac{1}{(2 \pi)^{N}} \mathcal{P}^{(N, a)}\{s\}\left[1+\mathrm{O}\left(\epsilon^{2}\right)\right],
$$

where

$$
\mathcal{P}^{(N, a)}\{s\}=\left(\prod_{l \in \mathcal{B}_{N}} \oint_{\mathcal{S}^{1}}\left|\mathrm{~d} \xi_{l}\right|\right) \mathcal{P}^{(N)}\{s, \xi\}
$$

is the $N$-mode amplitude PDF.

2. The amplitude variables are almost independent in a sense that for each $M \ll N$ modes, the $M$-mode amplitude PDF is equal to the product of the one-mode PDFs up to $\mathrm{O}(M / N)$ and $\mathrm{O}\left(\epsilon^{2}\right)$ corrections,

$$
\mathcal{P}_{j_{1}, j_{2}, \ldots, j_{M}}=P_{j_{1}}^{(a)} P_{j_{2}}^{(a)} \ldots P_{j_{M}}^{(a)}\left[1+\mathrm{O}(M / N)+\mathrm{O}\left(\epsilon^{2}\right)\right]
$$

\section{Weak nonlinearity and separation of time scales}

Consider weakly nonlinear dispersive waves in a periodic box. Here we consider quadratic nonlinearity and the linear dispersion relations $\omega_{k}$ which allow three-wave interactions. Example of such systems include surface capillary waves [5,12], Rossby waves [13] and internal waves in the ocean [14]. In Fourier space, we have the following Hamiltonian equations,

$$
i \dot{a}_{l}=\epsilon \sum_{m, n=1}^{\infty}\left(V_{m n}^{l} a_{m} a_{n} \mathrm{e}^{i \omega_{m n}^{l} t} \delta_{m+n}^{l}+2 \bar{V}_{l n}^{m} \bar{a}_{n} a_{m} \mathrm{e}^{-i \omega_{l n}^{m} t} \delta_{l+n}^{m}\right),
$$

where $a_{l}=a\left(k_{l}\right)$ is the complex wave amplitude in the interaction representation, $k_{l}=2 \pi l / L$ is the wavevector, $L$ is the box side length, $\omega_{m n}^{l} \equiv \omega_{k_{l}}-\omega_{k_{m}}-\omega_{k_{m}}, \omega_{l}=\omega_{k_{l}}$ is the wave frequency, $V_{m n}^{l}$ is an interaction coefficient and $\epsilon$ is a formal small nonlinearity parameter.

In order to filter out fast oscillations at the wave period, let us seek for the solution at time $T$ such that $2 \pi / \omega \ll$ $T \ll 1 / \omega \epsilon^{2}$. The second condition ensures that $T$ is a lot less than the nonlinear evolution time. Now let us use a perturbation expansion in small $\epsilon$,

$$
a_{l}(T)=a_{l}^{(0)}+\epsilon a_{l}^{(1)}+\epsilon^{2} a_{l}^{(2)} .
$$

Substituting this expansion in (7), we get in the zeroth order $a_{l}^{(0)}(T)=a_{l}(0)$, i.e., the zeroth order term is time independent. This corresponds to the fact that the interaction representation wave amplitudes are constant in the 
linear approximation. For simplicity, we will write $a_{l}^{(0)}(0)=a_{l}$, understanding that a quantity is taken at $t=0$ if its time argument is not mentioned explicitly. The first order is given by

$$
a_{l}^{(1)}(T)=-i \sum_{m, n=1}^{\infty}\left(V_{m n}^{l} a_{m} a_{n} \Delta_{m n}^{l} \delta_{m+n}^{l}+2 \bar{V}_{l n}^{m} a_{m} \bar{a}_{n} \bar{\Delta}_{l n}^{m} \delta_{l+n}^{m}\right),
$$

where $\Delta_{m n}^{l}=\int_{0}^{T} \mathrm{e}^{i \omega_{m n}^{l} t} \mathrm{~d} t=\left(\mathrm{e}^{i \omega_{m n}^{l} T}-1\right) / i \omega_{m n}^{l}$. Here we have taken into account that $a_{l}^{(0)}(T)=a_{l}$ and $a_{k}^{(1)}(0)=0$. Iterating one more time we get

$$
\begin{aligned}
a_{l}^{(2)}(T)= & \sum_{m, n, \mu, \nu}^{\infty}\left[2 V_{m n}^{l}\left(-V_{\mu \nu}^{m} a_{n} a_{\mu} a_{\nu} E\left[\omega_{n \mu \nu}^{l}, \omega_{m n}^{l}\right] \delta_{\mu+\nu}^{m}-2 \bar{V}_{m \nu}^{\mu} a_{n} a_{\mu} \bar{a}_{\nu} \bar{E}\left[\omega_{n \mu}^{l v}, \omega_{m n}^{l}\right] \delta_{m+v}^{\mu}\right) \delta_{m+n}^{l}\right. \\
& +2 \bar{V}_{l n}^{m}\left(-V_{\mu \nu}^{m} \bar{a}_{n} a_{\mu} a_{\nu} E\left[\omega_{\mu \nu}^{l n},-\omega_{l n}^{m}\right] \delta_{\mu+\nu}^{m}-2 \bar{V}_{m \nu}^{\mu} \bar{a}_{n} a_{\mu} \bar{a}_{\nu} E\left[-\omega_{n \nu l}^{\mu},-\omega_{l n}^{m}\right] \delta_{m+v}^{\mu}\right) \delta_{l+n}^{m} \\
& \left.+2 \bar{V}_{l n}^{m}\left(\bar{V}_{\mu \nu}^{n} a_{m} \bar{a}_{\mu} \bar{a}_{\nu} \delta_{\mu+\nu}^{n} E\left[-\omega_{l \nu \mu}^{m},-\omega_{l n}^{m}\right]+2 V_{n \nu}^{\mu} a_{m} \bar{a}_{\mu} a_{\nu} E\left[\omega_{v m}^{\mu l},-\omega_{l n}^{m}\right] \delta_{n+\nu}^{\mu}\right) \delta_{l+n}^{m}\right]
\end{aligned}
$$

where we used $a_{k}^{(2)}(0)=0$ and introduced $E(x, y)=\int_{0}^{T} \Delta(x-y) \mathrm{e}^{i y t} \mathrm{~d} t$.

\section{Evolution of the multi-mode PDF}

In this section we will apply the approach of $[19,20]$ to derive the evolution equation for the multi-mode PDF via introducing a generating functional, performing a weak-nonlinearity expansion and statistical averaging aided by a new graphical technique. We are going to demonstrate the phase independence property. This will also prepare us to answer the question of the next section: to what extent the amplitudes are going to remain statistically independent?

\subsection{Generating functional}

Introduction of generating functionals often simplifies statistical derivations but it can be defined differently to suit a particular technique. For our problem, the most useful form of the generating functional is

$$
Z^{(N)}\{\lambda, \mu\}=\frac{1}{(2 \pi)^{N}}\left\langle\prod_{l \in \mathcal{B}_{N}} \mathrm{e}^{\lambda_{l} A_{l}^{2}} \psi_{l}^{\mu_{l}}\right\rangle,
$$

where $\{\lambda, \mu\} \equiv\left\{\lambda_{l}, \mu_{l} ; l \in \mathcal{B}_{N}\right\}$ is a set of parameters, $\lambda_{l} \in \mathcal{R}$ and $\mu_{l} \in \mathcal{Z}$. Then:

$$
\mathcal{P}^{(N)}\{s, \xi\}=\frac{1}{(2 \pi)^{N}} \sum_{\{\mu\}}\left\langle\prod_{l \in \mathcal{B}_{N}} \delta\left(s_{l}-A_{l}^{2}\right) \psi_{l}^{\mu_{l}} \xi_{l}^{-\mu_{l}}\right\rangle,
$$

where $\{\mu\} \equiv\left\{\mu_{l} \in \mathcal{Z} ; l \in \mathcal{B}_{N}\right\}$. This expression can be verified by considering mean of a function $f\left\{A^{2}, \psi\right\}$ using the averaging rule (1) and expanding $f$ in the angular harmonics $\psi_{l}^{m} ; m \in \mathcal{Z}$ (basis functions on the unit circle),

$$
f\left\{A^{2}, \psi\right\}=\sum_{\{m\}} g\{m, A\} \prod_{l \in \mathcal{B}_{N}} \psi_{l}^{m_{l}}
$$

where $\{m\} \equiv\left\{m_{l} \in \mathcal{Z} ; l \in \mathcal{B}_{N}\right\}$ are indices enumerating the angular harmonics. Substituting this into (1) with PDF given by (12) and taking into account that any non-zero power of $\xi_{l}$ will give zero after the integration over the 
unit circle, one can see that LHS = RHS, i.e., that (12) is correct. Now we can easily represent (12) in terms of the generating functional,

$$
\mathcal{P}^{(N)}\{s, \xi\}=\hat{\mathcal{L}}_{\lambda}^{-1} \sum_{\{\mu\}}\left(Z^{(N)}\{\lambda, \mu\} \prod_{l \in \mathcal{B}_{N}} \xi_{l}^{-\mu_{l}}\right),
$$

where $\hat{\mathcal{L}}_{\lambda}^{-1}$ stands for inverse the Laplace transform with respect to all $\lambda_{l}$ parameters and $\{\mu\} \equiv\left\{\mu_{l} \in \mathcal{Z} ; l \in \mathcal{B}_{N}\right\}$ are the angular harmonics indices.

By definition, in RPA fields all variables $A_{l}$ and $\psi_{l}$ are statistically independent and $\psi_{l}$ 's are uniformly distributed on the unit circle. Such fields imply the following form of the generating functional

$$
Z^{(N)}\{\lambda, \mu\}=Z^{(N, a)}\{\lambda\} \prod_{l \in \mathcal{B}_{N}} \delta\left(\mu_{l}\right)
$$

where

$$
Z^{(N, a)}\{\lambda\}=\left\langle\prod_{l \in \mathcal{B}_{N}} \mathrm{e}^{\lambda_{l} A_{l}^{2}}\right\rangle=\left.Z^{(N)}\{\lambda, \mu\}\right|_{\mu=0}
$$

is an $N$-mode generating function for the amplitude statistics. Here, the Kronecker symbol $\delta\left(\mu_{l}\right)$ ensures independence of the PDF from the phase factors $\psi_{l}$. As a first step in validating the RPA property we will have to prove that the generating functional remains of form (15) up to $1 / N$ and $\mathrm{O}\left(\epsilon^{2}\right)$ corrections over the nonlinear time provided it has this form at $t=0$.

\subsection{Asymptotic expansion of the generating functional}

Let us first obtain an asymptotic weak-nonlinearity expansion for the generating functional $Z\{\lambda, \mu\}$ exploiting the separation of the linear and nonlinear time scales. ${ }^{2}$ To do this, we have to calculate $Z$ at the intermediate time $t=T$ via substituting into it $a_{j}(T)$ from (8) and retaining the terms up to $\mathrm{O}\left(\epsilon^{2}\right)$ only. This calculation is given in Appendix and the result of it is:

$$
Z\{\lambda, \mu, T\}=X\{\lambda, \mu, T\}+\bar{X}\{\lambda,-\mu, T\}
$$

with

$$
X\{\lambda, \mu, T\}=X(0)+(2 \pi)^{2 N}\left\langle\prod_{\|l\|<N} \mathrm{e}^{\lambda_{l}\left|a_{l}^{(0)}\right|^{2}}\left[\epsilon J_{1}+\epsilon^{2}\left(J_{2}+J_{3}+J_{4}+J_{5}\right)\right]\right\rangle_{A}+\mathrm{O}\left(\epsilon^{4}\right),
$$

where

$$
\begin{aligned}
& J_{1}=\left\langle\prod_{l} \psi_{l}^{(0) \mu_{l}} \sum_{j}\left(\lambda_{j}+\frac{\mu_{j}}{2\left|a_{j}^{(0)}\right|^{2}}\right) a_{j}^{(1)} \bar{a}_{j}^{(0)}\right\rangle_{\psi}, \\
& J_{2}=\frac{1}{2}\left\langle\prod_{l} \psi_{l}^{(0) \mu_{l}} \sum_{j}\left(\lambda_{j}+\lambda_{j}^{2}\left|a_{j}^{(0)}\right|^{2}-\frac{\mu_{j}^{2}}{2\left|a_{j}^{(0)}\right|^{2}}\right)\left|a_{j}^{(1)}\right|^{2}\right\rangle_{\psi},
\end{aligned}
$$

\footnotetext{
${ }^{2}$ Hereafter we omit superscript $(N)$ in the $N$-mode objects if it does not lead to a confusion.
} 


$$
\begin{gathered}
J_{3}=\left\langle\prod_{l} \psi_{l}^{(0) \mu_{l}} \sum_{j}\left(\lambda_{j}+\frac{\mu_{j}}{2\left|a_{j}^{(0)}\right|^{2}}\right) a_{j}^{(2)} \bar{a}_{j}^{(0)}\right\rangle_{\psi}, \\
J_{4}=\left\langle\prod_{l} \psi_{l}^{(0) \mu_{l}} \sum_{j}\left[\frac{\lambda_{j}^{2}}{2}+\frac{\mu_{j}}{4\left|a_{j}^{(0)}\right|^{4}}\left(\frac{\mu_{j}}{2}-1\right)+\frac{\lambda_{j} \mu_{j}}{2\left|a_{j}^{(0)}\right|^{2}}\right]\left(a_{j}^{(1)} \bar{a}_{j}^{(0)}\right)^{2}\right\rangle_{\psi}, \\
J_{5}=\frac{1}{2}\left\langle\prod_{l} \psi_{l}^{(0) \mu_{l}} \sum_{j \neq k} \lambda_{j} \lambda_{k}\left(a_{j}^{(1)} \bar{a}_{j}^{(0)}+\bar{a}_{j}^{(1)} a_{j}^{(0)}\right) a_{k}^{(1)} \bar{a}_{k}^{(0)}\right. \\
\left.+\left(\lambda_{j}+\frac{\mu_{j}}{4\left|a_{j}^{(0)}\right|^{2}}\right) \frac{\mu_{k}}{\left|a_{k}^{(0)}\right|^{2}}\left(a_{k}^{(1)} \bar{a}_{k}^{(0)}-\bar{a}_{k}^{(1)} a_{k}^{(0)}\right) a_{j}^{(1)} \bar{a}_{j}^{(0)}\right\rangle_{\psi},
\end{gathered}
$$

where $\langle\cdot\rangle_{A}$ and $\langle\cdot\rangle_{\psi}$ denote the averaging over the initial amplitudes and initial phases (which can be done independently). Our next step will be to calculate the above terms by substituting into them the values of $a^{(1)}$ and $a^{(2)}$ from (9) and (10), respectively.

\subsection{Statistical averaging and graphs}

Let us consider the initial fields $a_{k}(0)=a_{k}^{(0)}$ are essentially RPA as defined above. We will perform averaging over the statistics of the initial fields in order to obtain an evolution equations, first for $Z$ and then for the multi-mode PDF. The ultimate goal of this exercise is to prove that the wavefield remains of the essentially RPA type over the nonlinear time.

Let us introduce a graphical classification of the above terms which will allow us to simplify the statistical averaging and to understand which terms are dominant. We will only consider here contributions from $J_{1}$ and $J_{2}$ which will allow us to understand the basic method. Calculation of the rest of the terms, $J_{3}, J_{4}$ and $J_{5}$, follows the same principles and can be found in Appendix B. First, The linear in $\epsilon$ terms are represented by $J_{1}$ which, upon using (9), becomes

$$
J_{1}=\left\langle\prod_{l} \psi_{l}^{\mu_{l}} \sum_{j, m, n}\left(\lambda_{j}+\frac{\mu_{j}}{2 A_{j}^{2}}\right)\left(V_{m n}^{j} a_{m} a_{n} \Delta_{m n}^{j} \delta_{m+n}^{j}+2 \bar{V}_{j n}^{m} a_{m} \bar{a}_{n} \bar{\Delta}_{j n}^{m} \delta_{j+n}^{m}\right) \bar{a}_{j}\right\rangle_{\psi} .
$$

Hereafter we omit, for brevity of notation, the superscript (0) because no other superscripts will appear from now on.

Let us introduce some graphical notations for a simple classification of different contributions to this and to other (more lengthy) formulae that will follow. Combination $V_{m n}^{j} \delta_{m+n}^{j}$ will be marked by a vertex joining three lines with in-coming $j$ and out-coming $m$ and $n$ directions. Complex conjugate $\bar{V}_{m n}^{j} \delta_{m+n}^{j}$ will be drawn by the same vertex but with the opposite in-coming and out-coming directions. Presence of $a_{j}$ and $\bar{a}_{j}$ will be indicated by dashed lines pointing away and toward the vertex respectively. ${ }^{3}$ Thus, the two terms in formula (24) can be schematically

\footnotetext{
${ }^{3}$ This technique provides a useful classification method but not a complete mathematical description of the terms involved.
} 
represented as follows,

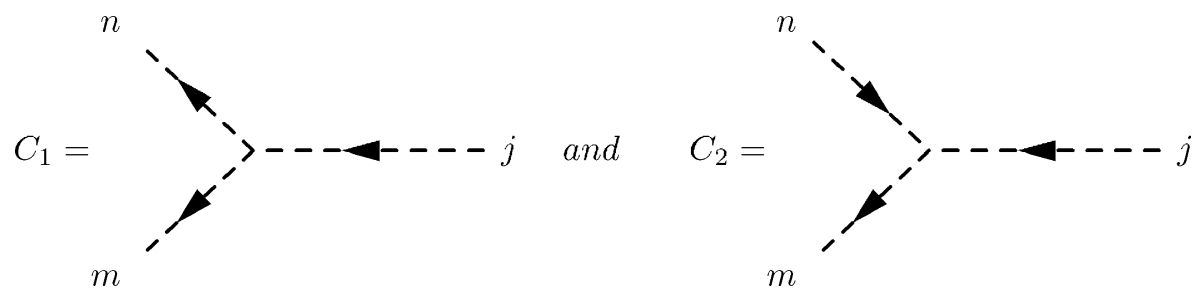

Let us average over all the independent phase factors in the set $\{\psi\}$. Such averaging takes into account the statistical independence and uniform distribution of variables $\psi$. In particular, $\langle\psi\rangle=0,\left\langle\psi_{l} \psi_{m}\right\rangle=0$ and $\left\langle\psi_{l} \bar{\psi}_{m}\right\rangle=$ $\delta_{l}^{m}$. Further, the products that involve odd number of $\psi$ 's are always zero, and among the even products only those can survive that have equal numbers of $\psi$ 's and $\bar{\psi}$ 's. These $\psi$ 's and $\bar{\psi}$ 's must cancel each other which is possible if their indices are matched in a pairwise way similarly to the Wick's theorem. The difference with the standard Wick, however, is that there exists possibility of not only internal (with respect to the sum) matchings but also external ones with $\psi$ 's in the pre-factor $\Pi \psi_{l}^{\mu_{l}}$.

Obviously, non-zero contributions can only arise for terms in which all $\psi$ 's cancel out either via internal mutual couplings within the sum or via their external couplings to the $\psi$ 's in the $l$-product. The internal couplings will indicate by joining the dashed lines into loops whereas the external matching will be shown as a dashed line pinned by a blob at the end. The number of blobs in a particular graph will be called the valence of this graph.

Note that there will be no contribution from the internal couplings between the incoming and the out-coming lines of the same vertex because, due to the $\delta$-symbol, one of the wavenumbers is 0 in this case, which means ${ }^{4}$ that $V=0$. For $J_{1}$ we have

$$
J_{1}=\left\langle C_{1}\right\rangle_{\psi}+\left\langle C_{2}\right\rangle_{\psi}
$$

with

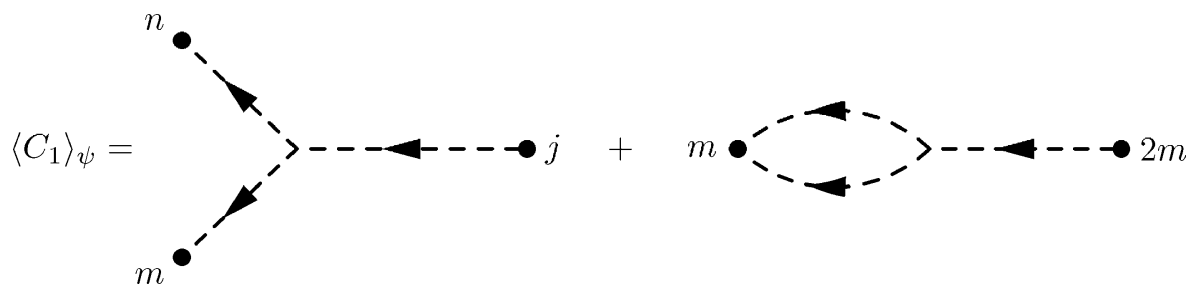

and

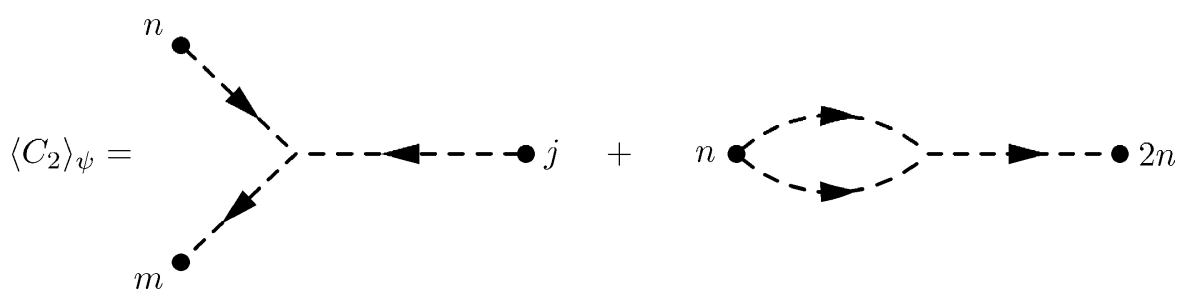

\footnotetext{
${ }^{4}$ In the present paper we consider only spatially homogeneous wave turbulence fields. In spatially homogeneous fields, due to momentum conservation, there is no coupling to the zero mode $k=0$ because such coupling would violate momentum conservation. Therefore, if one of the arguments of the interaction matrix element $V$ is equal to zero, the matrix element is identically zero. That is to say that for any spatially homogeneous wave turbulence system $V_{k_{1} k_{2}}^{k=0}=V_{k_{1}=0 k_{2}}^{k}=V_{k_{1} k_{2}=0}^{k}=0$.
} 
which correspond to the following expressions,

$$
\begin{aligned}
\left\langle C_{1}\right\rangle_{\psi}= & \sum_{j \neq m \neq n}\left(\lambda_{j}+\frac{\mu_{j}}{2 A_{j}^{2}}\right) V_{m n}^{j} A_{m} A_{n} A_{j} \Delta_{m n}^{j} \delta_{m+n}^{j} \delta\left(\mu_{m}+1\right) \delta\left(\mu_{n}+1\right) \delta\left(\mu_{j}-1\right) \prod_{l \neq j, m, n} \delta\left(\mu_{l}\right) \\
& +\sum_{m}\left(\lambda_{2 m}+\frac{\mu_{2 m}}{2 A_{2 m}^{2}}\right) V_{m m}^{2 m} A_{m}^{2} A_{2 m} \Delta_{m m}^{2 m} \delta\left(\mu_{m}+2\right) \delta\left(\mu_{2 m}-1\right) \prod_{l \neq m, 2 m} \delta\left(\mu_{l}\right)
\end{aligned}
$$

and

$$
\begin{aligned}
\left\langle C_{2}\right\rangle_{\psi}= & 2 \sum_{j \neq m \neq n}\left(\lambda_{j}+\frac{\mu_{j}}{2 A_{j}^{2}}\right) \bar{V}_{j n}^{m} A_{m} A_{n} A_{j} \bar{\Delta}_{j n}^{m} \delta_{j+n}^{m} \delta\left(\mu_{m}+1\right) \delta\left(\mu_{n}-1\right) \delta\left(\mu_{j}-1\right) \prod_{l \neq j, m, n} \delta\left(\mu_{l}\right) \\
& +2 \sum_{n}\left(\lambda_{n}+\frac{\mu_{n}}{2 A_{n}^{2}}\right) \bar{V}_{n n}^{2 n} A_{2 n} A_{n}^{2} \bar{\Delta}_{n n}^{2 n} \delta\left(\mu_{2 n}+1\right) \delta\left(\mu_{n}-2\right) \prod_{l \neq n, 2 n} \delta\left(\mu_{l}\right) .
\end{aligned}
$$

Because of the $\delta$-symbols involving $\mu$ 's, it takes very special combinations of the arguments $\mu$ in $Z\{\mu\}$ for the terms in the above expressions to be non-zero. For example, a particular term in the first sum of (25) may be non-zero if two $\mu$ 's in the set $\{\mu\}$ are equal to 1 whereas the rest of them are 0 . But in this case there is only one other term in this sum (corresponding to the exchange of values of $n$ and $j$ ) that may be non-zero too. In fact, only utmost two terms in the both (25) and (26) can be non-zero simultaneously. In the other words, each external pinning of the dashed line removes summation in one index and, since all the indices are pinned in the above diagrams, we are left with no summation at all in $J_{1}$, i.e., the number of terms in $J_{1}$ is $\mathrm{O}(1)$ with respect to large $N$. We will see later that the dominant contributions have $\mathrm{O}\left(N^{2}\right)$ terms. Although these terms come in the $\epsilon^{2}$ order, they will be much greater that the $\epsilon^{1}$ terms because the limit $N \rightarrow \infty$ must always be taken before $\epsilon \rightarrow 0$.

Let us consider the first of the $\epsilon^{2}$ terms, $J_{2}$. Substituting (9) into (20), we have

$$
\begin{aligned}
J_{2}= & \frac{1}{2}\left\langle\prod_{l} \psi_{l}^{(0) \mu_{l}} \sum_{j, m, n, \kappa, v}\left(\lambda_{j}+\lambda_{j}^{2} A_{j}^{2}-\frac{\mu_{j}^{2}}{2 A_{j}^{2}}\right) \times\left(V_{m n}^{j} a_{m} a_{n} \Delta_{m n}^{j} \delta_{m+n}^{j}+2 \bar{V}_{j n}^{m} a_{m} \bar{a}_{n} \bar{\Delta}_{j n}^{m} \delta_{j+n}^{m}\right)\right. \\
& \left.\times\left(\bar{V}_{\kappa \nu}^{j} \bar{a}_{\kappa} \bar{a}_{\nu} \bar{\Delta}_{\kappa \nu}^{j} \delta_{\kappa+\nu}^{j}+2 V_{j \nu}^{\kappa} \bar{a}_{\kappa} a_{\nu} \Delta_{j \nu}^{\kappa} \delta_{j+\nu}^{\kappa}\right)\right\rangle_{\psi}=\left\langle B_{1}+B_{2}+\bar{B}_{2}+B_{3}\right\rangle_{\psi},
\end{aligned}
$$

where
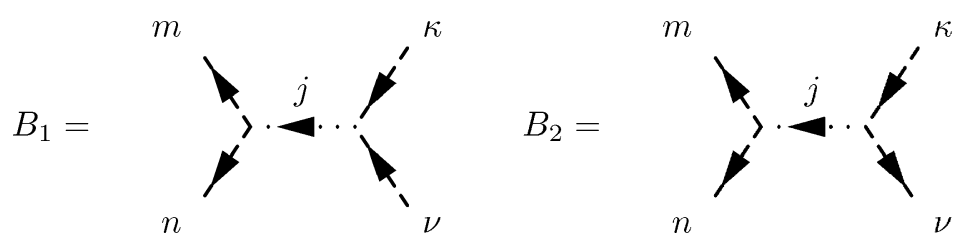

and $B_{3}=$

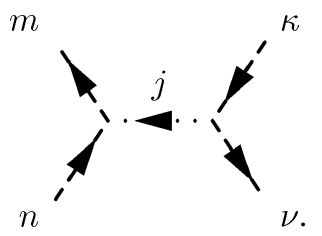

Here the graphical notation for the interaction coefficients $V$ and the amplitude $a$ is the same as introduced in the previous section and the dotted line with index $j$ indicates that there is a summation over $j$ but there is no amplitude $a_{j}$ in the corresponding expression. 
Let us now perform the phase averaging which corresponds to the internal and external couplings of the dashed lines. For $\left\langle B_{1}\right\rangle_{\psi}$ we have
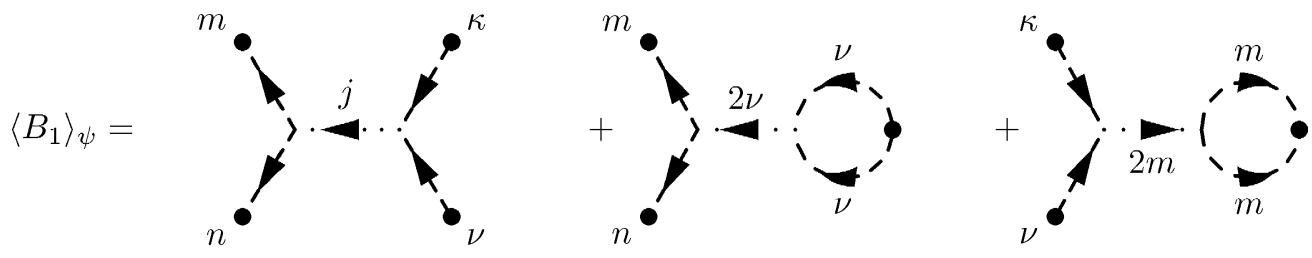

$+2$
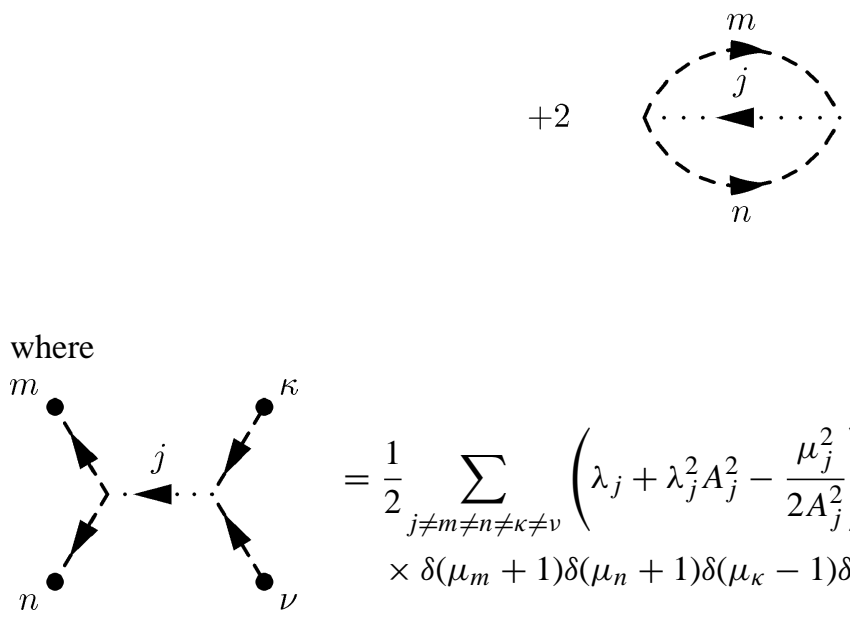

$$
\begin{aligned}
& =\frac{1}{2} \sum_{j \neq m \neq n \neq \kappa \neq v}\left(\lambda_{j}+\lambda_{j}^{2} A_{j}^{2}-\frac{\mu_{j}^{2}}{2 A_{j}^{2}}\right) V_{m n}^{j} \bar{V}_{\kappa v}^{j} \Delta_{m n}^{j} \bar{\Delta}_{\kappa \nu}^{j} \delta_{m+n}^{j} \delta_{\kappa+v}^{j} A_{m} A_{n} A_{\kappa} A_{v} \\
& \quad \times \delta\left(\mu_{m}+1\right) \delta\left(\mu_{n}+1\right) \delta\left(\mu_{\kappa}-1\right) \delta\left(\mu_{v}-1\right) \prod_{l \neq m, n, \kappa, v} \delta\left(\mu_{l}\right),
\end{aligned}
$$

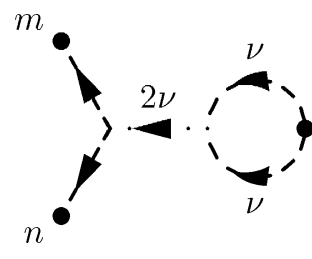

$$
\begin{aligned}
= & \frac{1}{2} \sum_{m \neq n \neq v}\left(\lambda_{2 v}+\lambda_{2 v}^{2} A_{2 v}^{2}-\frac{\mu_{2 v}^{2}}{2 A_{2 v}^{2}}\right) V_{m n}^{2 v} \delta_{m+n}^{2 v} \bar{V}_{\kappa v}^{2 v} \\
& \Delta_{m n}^{2 v} \bar{\Delta}_{\kappa v}^{2 v} A_{m} A_{n} A_{v}^{2} \delta\left(\mu_{m}+1\right) \delta\left(\mu_{n}+1\right) \delta\left(\mu_{v}-2\right) \prod_{l \neq m, n, v} \delta\left(\mu_{l}\right),
\end{aligned}
$$<smiles>c1ccc2ccccc2c1</smiles>

$$
=\prod_{l} \delta\left(\mu_{l}\right) \sum_{j, m, n}\left(\lambda_{j}+\lambda_{j}^{2} A_{j}^{2}\right)\left|V_{m n}^{j}\right|^{2}\left|\Delta_{m n}^{j}\right|^{2} \delta_{m+n}^{j} A_{m}^{2} A_{n}^{2} .
$$

We have not written out the third term in (29) because it is just a complex conjugate of the second one. Observe that all the diagrams in the first line of (29) are $\mathrm{O}(1)$ with respect to large $N$ because all of the summations are lost due to the external couplings (compare with the previous section). On the other hand, the diagram in the second line contains two purely-internal couplings and is therefore $\mathrm{O}\left(N^{2}\right)$. This is because the number of indices over which the summation survives is equal to the number of purely internal couplings. Thus, the zero-valent graphs are dominant and we can write

$$
\left\langle B_{1}\right\rangle_{\psi}=\prod_{l} \delta\left(\mu_{l}\right) \sum_{j, m, n}\left(\lambda_{j}+\lambda_{j}^{2} A_{j}^{2}\right)\left|V_{m n}^{j}\right|^{2}\left|\Delta_{m n}^{j}\right|^{2} \delta_{m+n}^{j} A_{m}^{2} A_{n}^{2}\left[1+\mathrm{O}\left(1 / N^{2}\right)\right] .
$$


For $\left\langle B_{2}\right\rangle_{\psi}$ we have

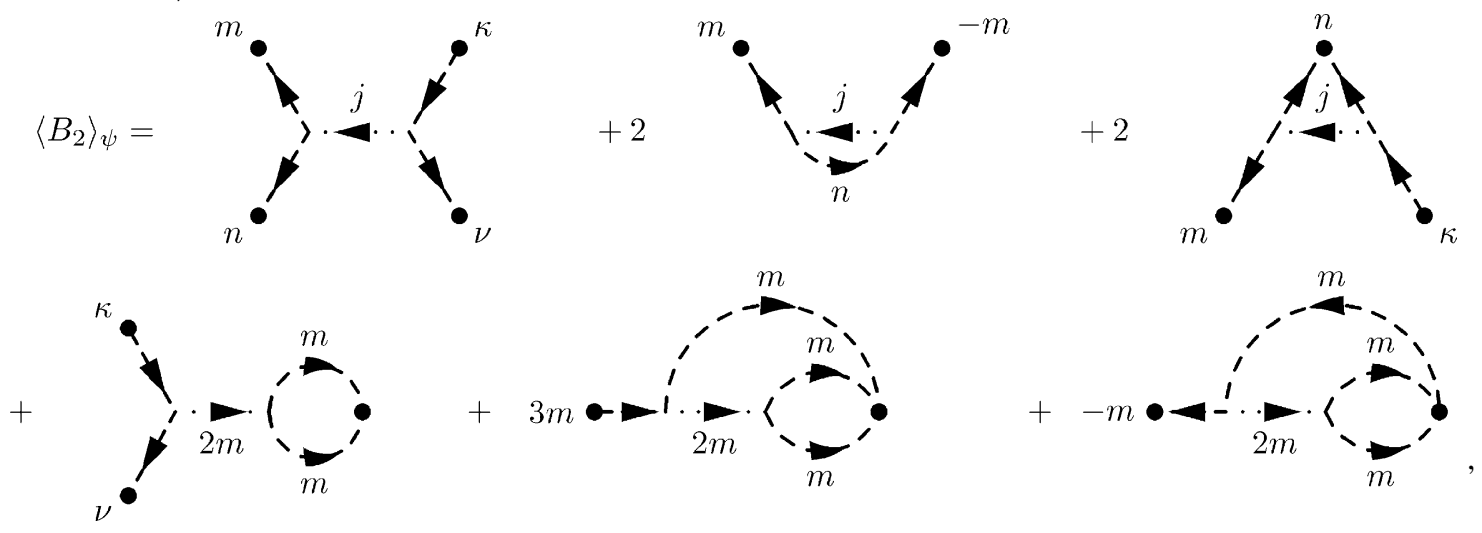

where

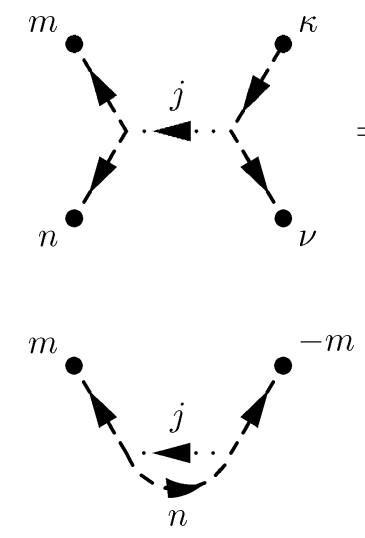

$$
\begin{array}{r}
=\sum_{j \neq m \neq n \neq \kappa \neq \nu}\left(\lambda_{j}+\lambda_{j}^{2} A_{j}^{2}-\frac{\mu_{j}^{2}}{2 A_{j}^{2}}\right) V_{m n}^{j} V_{j \nu}^{\kappa} \Delta_{m n}^{j} \Delta_{j \nu}^{\kappa} \delta_{m+n}^{j} \delta_{j+\nu}^{\kappa} A_{m} A_{n} A_{\kappa} A_{\nu} \\
\times \delta\left(\mu_{m}+1\right) \delta\left(\mu_{n}+1\right) \delta\left(\mu_{\kappa}-1\right) \delta\left(\mu_{\nu}+1\right) \prod_{l \neq m, n, \kappa, \nu} \delta\left(\mu_{l}\right)
\end{array}
$$$$
=\sum_{j, m, n}\left(\lambda_{j}+\lambda_{j}^{2} A_{j}^{2}-\frac{\mu_{j}^{2}}{2 A_{j}^{2}}\right) V_{m n}^{j} V_{j-m}^{n} \Delta_{m n}^{j}
$$$$
\times \Delta_{j-m}^{n} \delta_{m+n}^{j} A_{n}^{2} A_{m} A_{-m} \delta\left(\mu_{m}+1\right) \delta\left(\mu_{-m}+1\right) \prod_{l \neq m,-m} \delta\left(\mu_{l}\right)
$$

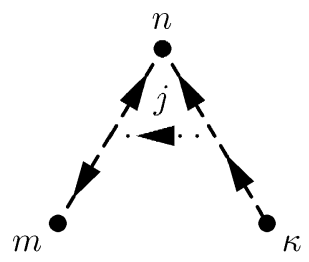

$$
\begin{aligned}
= & \sum_{j \neq m \neq n \neq \kappa}\left(\lambda_{j}+\lambda_{j}^{2} A_{j}^{2}-\frac{\mu_{j}^{2}}{2 A_{j}^{2}}\right) V_{m n}^{j} V_{j n}^{\kappa} \\
& \times \Delta_{m n}^{j} \Delta_{j n}^{\kappa} \delta_{m+n}^{j} \delta_{j+n}^{\kappa} A_{m} A_{n}^{2} A_{\kappa} \delta\left(\mu_{m}+1\right) \delta\left(\mu_{n}+2\right) \delta\left(\mu_{\kappa}-1\right) \prod_{l \neq m, n, \kappa} \delta\left(\mu_{l}\right)
\end{aligned}
$$

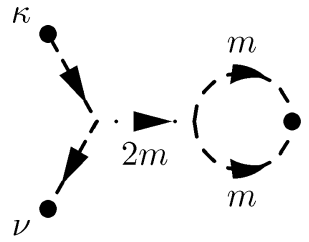

$$
\begin{aligned}
&= \sum_{m \neq \kappa \neq \nu}\left(\lambda_{2 m}+\lambda_{2 m}^{2} A_{2 m}^{2}-\frac{\mu_{2 m}^{2}}{2 A_{2 m}^{2}}\right) V_{m m}^{2 m} V_{2 m \nu}^{\kappa} \\
& \times \Delta_{m m}^{2 m} \Delta_{2 m \nu}^{\kappa} \delta_{2 m+\nu}^{\kappa} A_{m}^{2} A_{\kappa} A_{\nu} \\
& \quad \times \delta\left(\mu_{m}+2\right) \delta\left(\mu_{\kappa}-1\right) \delta\left(\mu_{\nu}+1\right) \prod_{l \neq m, \kappa, \nu} \delta\left(\mu_{l}\right)
\end{aligned}
$$




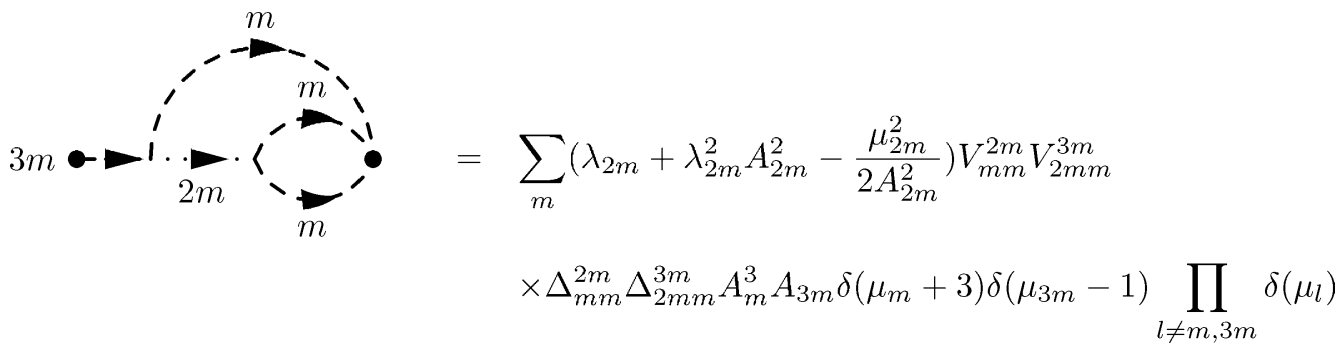

$\times \Delta_{2 m-m}^{m} A_{m}^{3} A_{-m} \delta\left(\mu_{m}+1\right) \delta\left(\mu_{-m}+1\right) \prod_{l \neq m,-m} \delta\left(\mu_{l}\right)$
$2 m=\lambda_{m}\left(\lambda_{2 m}+\lambda_{2 m}^{2} A_{2 m}^{2}-\frac{\mu_{2 m}^{2}}{2 A_{2 m}^{2}}\right) V_{m m}^{2 m} V_{2 m-m}^{m} \Delta_{m m}^{2 m}$

The second term in (31) contains one summation because its graph has one purely internal coupling. This term is $N$ times smaller than the largest terms in $\left\langle B_{1}\right\rangle_{\psi}$ (which have 2 surviving summation indices). All the other terms in (31) contain no summation at all because all their dashed lines are coupled externally.

Similarly, the leading contribution to $\left\langle B_{3}\right\rangle_{\psi}$ will be given by the zero-valent graph with the maximum possible number of internal couplings (which is equal to 2 in this case). Because of the $\delta$ 's, there are no graphs with just one internal coupling, but there are graphs with all the dashed lines coupled externally. Thus,

$$
\begin{aligned}
\left\langle B_{3}\right\rangle_{\psi}= & \\
= & =\prod_{l} \delta\left(\mu_{l}\right) \sum_{j, m, n}\left(\lambda_{j}+\lambda_{j}^{2} A_{j}^{2}\right)\left|V_{j n}^{m}\right|^{2}\left|\Delta_{j n}^{m}\right|^{2} \delta_{j+n}^{m} A_{m}^{2} A_{n}^{2}\left[1+O\left(1 / N^{2}\right)\right],
\end{aligned}
$$

Summarising the results of this section we can write for $J_{2}$ :

$$
J_{2}=\prod_{l} \delta\left(\mu_{l}\right) \sum_{j, m, n}\left(\lambda_{j}+\lambda_{j}^{2} A_{j}^{2}\right)\left[\left|V_{m n}^{j}\right|^{2}\left|\Delta_{m n}^{j}\right|^{2} \delta_{m+n}^{j}+2\left|V_{j n}^{m}\right|^{2}\left|\Delta_{j n}^{m}\right|^{2} \delta_{j+n}^{m}\right] A_{m}^{2} A_{n}^{2}[1+\mathrm{O}(1 / N)] .
$$

Thus, we considered in detail the different terms involved in $J_{2}$ and we found that the dominant contributions come from the zero-valent graphs because the have more summation indices involved. This turns out to be the general rule that allows one to simplify calculation by discarding a significant number of graphs with non-zero valence. After this observation finding the rest of the terms, $J_{3}$ to $J_{5}$, becomes a routine task and we therefore move it to the Appendix B.

\subsection{Equation for $Z$}

Now we can observe that all contributions to the evolution of $Z$ (namely $J_{1}-J_{5}$, see the previous section and Appendix 2) contain factor $\prod_{l} \delta\left(\mu_{l}\right)$ which means that the phase factors $\{\psi\}$ remain a set of statistically independent (of each other and of $A$ 's) variables uniformly distributed on $S^{1}$. This is true with accuracy $\mathrm{O}\left(\epsilon^{2}\right)$ (assuming that 
the $N$-limit is taken first, i.e., $1 / N \ll \epsilon^{2}$ ) and this proves persistence of the first of the "essential RPA" properties. Similar result for a special class of three-wave systems arising in the solid state physics was previously obtained by Brout and Prigogine [16]. This result is interesting because it has been obtained without any assumptions on the statistics of the amplitudes $\{A\}$ and, therefore, it is valid beyond the RPA approach. It may appear useful in future for study of fields with random phases but correlated amplitudes.

Let us now derive an evolution equation for the generating functional. Using our results for $J_{1}-J_{5}$ in (18) and (17) we have

$$
\begin{aligned}
Z(T)-Z(0)= & \epsilon^{2} \sum_{j, m, n}\left(\lambda_{j}+\lambda_{j}^{2} \frac{\partial}{\partial \lambda_{j}}\right)\left[\left|V_{m n}^{j}\right|^{2}\left|\Delta_{m n}^{j}\right|^{2} \delta_{m+n}^{j}+2\left|V_{j n}^{m}\right|^{2}\left|\Delta_{j n}^{m}\right|^{2} \delta_{j+n}^{m}\right] \frac{\partial^{2} Z(0)}{\partial \lambda_{m} \partial \lambda_{n}} \\
& +4 \epsilon^{2} \sum_{j, m, n} \lambda_{j}\left[-\left|V_{m n}^{j}\right|^{2} \bar{E}\left(0, \omega_{m n}^{j}\right) \delta_{m+n}^{j} \frac{\partial}{\partial \lambda_{n}}+\left|V_{j n}^{m}\right|^{2} E\left(0,-\omega_{j n}^{m}\right) \delta_{j+n}^{m}\left(\frac{\partial}{\partial \lambda_{m}}-\frac{\partial}{\partial \lambda_{n}}\right)\right] \\
& \times \frac{\partial Z(0)}{\partial \lambda_{j}}+2 \epsilon^{2} \sum_{j \neq k, n} \lambda_{j} \lambda_{k}\left[-2\left|V_{k n}^{j}\right|^{2} \delta_{k+n}^{j}\left|\Delta_{k n}^{j}\right|^{2}+\left|V_{j k}^{n}\right|^{2} \delta_{j+k}^{n}\left|\Delta_{j k}^{n}\right|^{2}\right] \frac{\partial^{3} Z(0)}{\partial \lambda_{j} \partial \lambda_{n} \partial \lambda_{k}}+c c .
\end{aligned}
$$

Here partial derivatives with respect to $\lambda_{l}$ appeared because of the $A_{l}$ factors. This expression is valid up to $\mathrm{O}\left(\epsilon^{4}\right)$ and $\mathrm{O}\left(\epsilon^{2} / N\right)$ corrections. Note that we still have not used any assumption about the statistics of $A$ 's. This is a linear equation: as usual in statistics we traded nonlinearity for higher dimensions. The last term here "spoils" the separation of variables and, therefore, puts a question mark on the independence of variables $\{A\}$ from each other on the nonlinear time.

Let us now $N \rightarrow \infty$ limit followed by $T \sim 1 / \epsilon \rightarrow \infty$ (we re-iterate that this order of the limits is essential). Taking into account that $\lim _{T \rightarrow \infty} E(0, x)=T(\pi \delta(x)+i P(1 / x))$, and $\lim _{T \rightarrow \infty}|\Delta(x)|^{2}=2 \pi T \delta(x)$ and, replacing $(Z(T)-Z(0)) / T$ by $\dot{Z}$ we have

$$
\begin{aligned}
\dot{Z}= & 4 \pi \epsilon^{2} \int\left\{\left(\lambda_{j}+\lambda_{j}^{2} \frac{\delta}{\delta \lambda_{j}}\right)\left[\left|V_{m n}^{j}\right|^{2} \delta\left(\omega_{m n}^{j}\right) \delta_{m+n}^{j}+2\left|V_{j n}^{m}\right|^{2} \delta\left(\omega_{j n}^{m}\right) \delta_{j+n}^{m}\right] \frac{\delta^{2} Z}{\delta \lambda_{m} \delta \lambda_{n}}\right. \\
& +2 \lambda_{j}\left[-\left|V_{m n}^{j}\right|^{2} \delta\left(\omega_{m n}^{j}\right) \delta_{m+n}^{j} \frac{\delta}{\delta \lambda_{n}}+\left|V_{j n}^{m}\right|^{2} \delta\left(\omega_{j n}^{m}\right) \delta_{j+n}^{m}\left(\frac{\delta}{\delta \lambda_{m}}-\frac{\delta}{\delta \lambda_{n}}\right)\right] \frac{\delta Z}{\delta \lambda_{j}} \\
& \left.+2 \lambda_{j} \lambda_{m}\left[-2\left|V_{m n}^{j}\right|^{2} \delta_{m+n}^{j} \delta\left(\omega_{m n}^{j}\right)+\left|V_{j m}^{n}\right|^{2} \delta_{j+m}^{n} \delta\left(\omega_{j m}^{n}\right)\right] \frac{\delta^{3} Z}{\delta \lambda_{j} \delta \lambda_{n} \delta \lambda_{m}}\right\} \mathrm{d} k_{j} \mathrm{~d} k_{m} \mathrm{~d} k_{n} .
\end{aligned}
$$

Here variational derivatives appeared instead of partial derivatives because of the $N \rightarrow \infty$ limit.

\subsection{Equation for the PDF}

Taking the inverse Laplace transform of (35) we have the following equation for the PDF,

$$
\dot{\mathcal{P}}=-\int \frac{\delta F_{j}}{\delta s_{j}} \mathrm{~d} k_{j}
$$

where $F_{j}$ is a flux of probability in the space of the amplitude $s_{j}$,

$$
\begin{aligned}
F_{j}= & 4 \pi \epsilon^{2} \int\left\{\left(\left|V_{m n}^{j}\right|^{2} \delta\left(\omega_{m n}^{j}\right) \delta_{m+n}^{j}+2\left|V_{j m}^{n}\right|^{2} \delta\left(\omega_{j m}^{n}\right) \delta_{j+m}^{n}\right)\left[s_{n} s_{m} \mathcal{P}-\frac{\delta}{\delta s_{j}}\left(s_{j} s_{n} s_{m} \mathcal{P}\right)\right]\right. \\
& -2 \mathcal{P}\left[\left|V_{m n}^{j}\right|^{2} \delta\left(\omega_{m n}^{j}\right) \delta_{m+n}^{j} s_{j} s_{m}+\left|V_{j m}^{n}\right|^{2} \delta\left(\omega_{j m}^{n}\right) \delta_{j+m}^{n}\left(s_{j} s_{m}-s_{j} s_{n}\right)\right] \\
& \left.-2\left(\left|V_{j m}^{n}\right|^{2} \delta\left(\omega_{j m}^{n}\right) \delta_{j+m}^{n}-2\left|V_{m n}^{j}\right|^{2} \delta\left(\omega_{m n}^{j}\right) \delta_{m+n}^{j}\right) \frac{\delta}{\delta s_{m}}\left(s_{j} s_{n} s_{m} \mathcal{P}\right)\right\} \mathrm{d} k_{m} \mathrm{~d} k_{n} .
\end{aligned}
$$


This expression can be simplified to

$$
\begin{aligned}
-\frac{F_{j}}{4 \pi \epsilon^{2} s_{j}}= & \int\left\{\left(\left|V_{m n}^{j}\right|^{2} \delta\left(\omega_{m n}^{j}\right) \delta_{m+n}^{j}+2\left|V_{j m}^{n}\right|^{2} \delta\left(\omega_{j m}^{n}\right) \delta_{j+m}^{n}\right) s_{n} s_{m} \frac{\delta \mathcal{P}}{\delta s_{j}}\right. \\
& +2 \mathcal{P}\left(\left|V_{j m}^{n}\right|^{2} \delta\left(\omega_{j m}^{n}\right) \delta_{j+m}^{n}-\left|V_{m n}^{j}\right|^{2} \delta\left(\omega_{m n}^{j}\right) \delta_{m+n}^{j}\right) s_{m}+2\left(\left|V_{j m}^{n}\right|^{2} \delta\left(\omega_{j m}^{n}\right) \delta_{j+m}^{n}\right. \\
& \left.\left.-2\left|V_{m n}^{j}\right|^{2} \delta\left(\omega_{m n}^{j}\right) \delta_{m+n}^{j}\right) s_{n} s_{m} \frac{\delta \mathcal{P}}{\delta s_{m}}\right\} \mathrm{d} k_{m} \mathrm{~d} k_{n} .
\end{aligned}
$$

This equation is identical to the one originally obtained by Peierls [15] and later rediscovered by Brout and Prigogine [16] in the context of the physics of anharmonic crystals. Zaslavskii and Sagdeev [17] were the first to study this equation in the WT context. However, the analysis of [15-17] was restricted to the interaction Hamiltonians of the "potential energy" type, i.e., the ones that involve only the coordinates but not the momenta. This restriction leaves aside a great many important WT systems, e.g., the capillary, Rossby, internal and MHD waves. Our result above indicates that the Peierls equation is also valid in the most general case of three-wave systems.

Here we should again emphasise importance of the taken order of limits, $N \rightarrow \infty$ first and $\epsilon \rightarrow 0$ second. Physically this means that the frequency resonance is broad enough to cover great many modes. Some authors, e.g., [15-17], leave the sum notation in the PDF equation even after the $\epsilon \rightarrow 0$ limit taken giving $\delta\left(\omega_{j m}^{n}\right)$. One has to be careful interpreting such formulae because formally the RHS is nil in most of the cases because there may be no exact resonances between the discrete $k$ modes (as it is the case, e.g., for the capillary waves). In real finite-size physical systems, this condition means that the wave amplitudes, although small, should not be too small so that the frequency broadening is sufficient to allow the resonant interactions. Our functional integral notation is meant to indicate that the $N \rightarrow \infty$ limit has already been taken.

\section{Approximate independence of the amplitudes}

Variables $s_{j}$ do not separate in the above equation for the PDF. Indeed, substituting

$$
\mathcal{P}^{(N, a)}=P_{j_{1}}^{(a)} P_{j_{2}}^{(a)} \ldots P_{j_{N}}^{(a)}
$$

into the discrete version of (38) we see that it turns into zero on the thermodynamic solution with $P_{j}^{(a)}=$ $\omega_{j} \exp \left(-\omega_{j} s_{j}\right)$. However, it is not zero for the one-mode PDF $P_{j}^{(a)}$ corresponding to the cascade-type KolmogorovZakharov (KZ) spectrum $n_{j}^{k z}$, i.e., $P_{j}^{(a)}=\left(1 / n_{j}^{k z}\right) \exp \left(-s_{j} / n_{j}^{k z}\right)$ (see next section), nor it is likely to be zero for any other PDF of form (39). This means that, even initially independent, the amplitudes will correlate with each other at the nonlinear time. Does this mean that the existing WT theory, and in particular the kinetic equation, is invalid?

To answer to this question let us differentiate the discrete version of the Eq. (35) with respect to $\lambda$ 's to get equations for the amplitude moments. We can easily see that

$$
\partial_{t}\left(\left\langle A_{j_{1}}^{2} A_{j_{2}}^{2}\right\rangle-\left\langle A_{j_{1}}^{2}\right\rangle\left\langle A_{j_{2}}^{2}\right\rangle\right)=\mathrm{O}\left(\epsilon^{4}\right) \quad\left(j_{1}, j_{2} \in \mathcal{B}_{N}\right)
$$

if $\left\langle A_{j_{1}}^{2} A_{j_{2}}^{2} A_{j_{3}}^{2}\right\rangle=\left\langle A_{j_{1}}^{2}\right\rangle\left\langle A_{j_{2}}^{2}\right\rangle\left\langle A_{j_{3}}^{2}\right\rangle$ (with the same accuracy) at $t=0$. Similarly, in terms of PDFs

$$
\partial_{t}\left(P_{j_{1}, j_{2}}^{(2, a)}\left(s_{j_{1}}, s_{j_{2}}\right)-P_{j_{1}}^{(a)}\left(s_{j_{1}}\right) P_{j_{2}}^{(a)}\left(s_{j_{2}}\right)\right)=\mathrm{O}\left(\epsilon^{4}\right) \quad\left(j_{1}, j_{2} \in \mathcal{B}_{N}\right),
$$

if $P_{j_{1}, j_{2}, j_{3}, j_{4}}^{(4, a)}\left(s_{j_{1}}, s_{j_{2}}, s_{j_{3}}, s_{j_{4}}\right)=P_{j_{1}}^{(a)}\left(s_{j_{1}}\right) P_{j_{2}}^{(a)}\left(s_{j_{2}}\right) P_{j_{3}}^{(a)}\left(s_{j_{3}}\right) P_{j_{4}}^{(a)}\left(s_{j_{4}}\right)$ at $t=0$. Here $P_{j_{1}, j_{2}, j_{3}, j_{4}}^{(4, a)}\left(s_{j_{1}}, s_{j_{2}}, s_{j_{3}}, s_{j_{4}}\right)$, $P_{j_{1}, j_{2}}^{(2, a)}\left(s_{j_{1}}, s_{j_{2}}\right)$ and $P_{j}^{(a)}\left(s_{j}\right)$ are the four-mode, two-mode and one-mode PDF's obtained from $\mathcal{P}$ by integrating out all but 3, 2 or 1 arguments, respectively. One can see that, with a $\epsilon^{2}$ accuracy, the Fourier modes will re- 
main independent of each other in any pair over the nonlinear time if they were independent in every triplet at $t=0$.

Similarly, one can show that the modes will remain independent over the nonlinear time in any subset of $M<N$ modes with accuracy $M / N$ (and $\epsilon^{2}$ ) if they were initially independent in every subset of size $M+1$. Namely

$$
P_{j_{1}, j_{2}, \ldots, j_{M}}^{(M, a)}\left(s_{j_{1}}, s_{j_{2}}, s_{j_{M}}\right)-P_{j_{1}}^{(a)}\left(s_{j_{1}}\right) P_{j_{2}}^{(a)}\left(s_{j_{2}}\right) \ldots P_{j_{M}}^{(a)}\left(s_{j_{M}}\right)=\mathrm{O}(M / N)+\mathrm{O}\left(\epsilon^{2}\right) \quad\left(j_{1}, j_{2}, \ldots, j_{M} \in \mathcal{B}_{N}\right)
$$

if $P_{j_{1}, j_{2}, \ldots, j_{M+1}}^{(M+1, a)}=P_{j_{1}}^{(a)} P_{j_{2}}^{(a)} \ldots P_{j_{M+1}}^{(a)}$ at $t=0$.

Mismatch $\mathrm{O}(M / N)$ arises from some terms in the ZS equation with coinciding indices $j$. For $M=2$ there is only one such term in the $N$-sum and, therefore, the corresponding error is $\mathrm{O}(1 / N)$ which is much less than $\mathrm{O}\left(\epsilon^{2}\right)$ (due to the order of the limits in $N$ and $\epsilon$ ). However, the number of such terms grows as $M$ and the error accumulates to $\mathrm{O}(M / N)$ which can greatly exceed $\mathrm{O}\left(\epsilon^{2}\right)$ for sufficiently large $M$.

We see that the accuracy with which the modes remain independent in a subset is worse for larger subsets and that the independence property is completely lost for subsets approaching in size the entire set, $M \sim N$. One should not worry too much about this loss because $N$ is the biggest parameter in the problem (size of the box) and the modes will be independent in all $M$-subsets no matter how large. Thus, the statistical objects involving any finite number of modes are factorisable as products of the one-mode objects and, therefore, the WT theory reduces to considering the one-mode objects. This results explains why we re-defined RPA in its relaxed "essential RPA" form. Indeed, in this form RPA is sufficient for the WT closure and, on the other hand, it remains valid over the nonlinear time. In particular, only property (40) is needed, as far as the amplitude statistics is concerned, for deriving the three-wave kinetic equation, and this fact validates this equation and all of its solutions, including the $\mathrm{KZ}$ spectrum which plays an important role in WT.

The situation were modes can be considered as independent when taken in relatively small sets but should be treated as dependent in the context of much larger sets is not so unusual in physics. Consider for example a distribution of electrons and ions in plasma. The full $N$-particle distribution function in this case satisfies the Liouville equation which is, in general, not a separable equation. In other words, the $N$-particle distribution function cannot be written as a product of $N$ one-particle distribution functions. However, an $M$-particle distribution can indeed be represented as a product of $M$ one-particle distributions if $M \ll N_{\mathrm{D}}$, where $N_{\mathrm{D}}$ is the number of particles in the Debye sphere. We see an interesting transition from a an individual to collective behaviour when the number of particles approaches $N_{\mathrm{D}}$. In the special case of the one-particle function we have here the famous mean-field Vlasov equation which is valid up to $\mathrm{O}\left(1 / N_{\mathrm{D}}\right)$ corrections (representing particle collisions).

\section{One-mode statistics}

We have established above that the one-point statistics is at the heart of the WT theory. All one-point statistical objects can be derived from the one-point amplitude generating function,

$$
Z_{a}\left(\lambda_{j}\right)=\left\langle\mathrm{e}^{\lambda_{j} A_{j}^{2}}\right\rangle
$$

which can be obtained from the $N$-point $Z$ by taking all $\mu$ 's and all $\lambda$ 's, except for $\lambda_{j}$, equal to zero. Substituting such values to (35) we get the following equation for $Z_{a}$,

$$
\frac{\partial Z_{a}}{\partial t}=\lambda_{j} \eta_{j} Z_{a}+\left(\lambda_{j}^{2} \eta_{j}-\lambda_{j} \gamma_{j}\right) \frac{\partial Z_{a}}{\partial \lambda_{j}},
$$


where,

$$
\begin{aligned}
& \eta_{j}=4 \pi \epsilon^{2} \int\left(\left|V_{l m}^{j}\right|^{2} \delta_{l m}^{j} \delta\left(\omega_{l m}^{j}\right)+2\left|V_{j l}^{m}\right|^{2} \delta_{j l}^{m} \delta\left(\omega_{j l}^{m}\right)\right) n_{l} n_{m} \mathrm{~d} k_{l} \mathrm{~d} k_{m}, \\
& \gamma_{j}=8 \pi \epsilon^{2} \int\left(\left|V_{l m}^{j}\right|^{2} \delta_{l m}^{j} \delta\left(\omega_{l m}^{j}\right) n_{m}+\left|V_{j l}^{m}\right|^{2} \delta_{j l}^{m} \delta\left(\omega_{j l}^{m}\right)\left(n_{l}-n_{m}\right)\right) \mathrm{d} k_{l} \mathrm{~d} k_{m} .
\end{aligned}
$$

Correspondingly, for the one mode $\operatorname{PDF} P_{a}\left(s_{j}\right)$ we have

$$
\frac{\partial P_{a}}{\partial t}+\frac{\partial F}{\partial s_{j}}=0
$$

with $F$ is a probability flux in the $s$-space,

$$
F=-s_{j}\left(\gamma P_{a}+\eta_{j} \frac{\delta P_{a}}{\delta s_{j}}\right)
$$

Eqs. (43) and (46) where previously obtained and studied in [20] in for the four-wave systems. The only difference for the four-wave case was different expressions for $\eta$ and $\gamma$. For the three-wave case, equation for the PDF was not considered before, but equations for its moments were derived and solved in [19]. In particular, equation for the first moment is nothing but the familiar kinetic equation $\dot{n}=-\gamma n+\eta$ which gives $\eta=\gamma n$ for any steady state. This, in turn means that in the steady state with $F=0$ we have $P_{j}^{(a)}=\left(1 / n_{j}\right) \exp \left(-s_{j} / n_{j}\right)$, where $n_{j}$ can be any steady state solution of the kinetic equation including the $\mathrm{KZ}$ spectrum which plays the central role in WT [5,1]. However, it was shown in [20] that there also exist solutions with $F \neq 0$ which describe WT intermittency.

\section{Phase statistics}

Importantly, RPA formulation involves independent phase factors $\psi=\mathrm{e}^{\phi}$ and not phases $\phi$ themselves. Firstly, the phases would not be convenient because, as we will see later, the mean value of the phases is evolving and one could not say that they are "distributed uniformly from $-\pi$ to $\pi$ ". In fact, we will also see that the mean fluctuation of the phase distribution is also growing and they quickly spread beyond their initial $2 \pi$-wide interval. But perhaps even more important, $\phi$ 's build mutual correlations on the nonlinear time whereas $\psi$ 's remain independent. This will be shown later in this section, but we would like first to give a simple example illustrating how this property is possible due to the fact that correspondence between $\phi$ and $\psi$ is not a bijection.

Let $N$ be a random integer and let $r_{1}$ and $r_{2}$ be two independent (of $N$ and of each other) random numbers with uniform distribution between $-\pi$ and $\pi$. Let

$$
\phi_{1,2}=2 \pi N+r_{1,2} .
$$

Then

$$
\left\langle\phi_{1,2}\right\rangle=2 \pi\langle N\rangle,
$$

and

$$
\left\langle\phi_{1} \phi_{2}\right\rangle=4 \pi^{2}\left\langle N^{2}\right\rangle .
$$


Thus,

$$
\left\langle\phi_{1} \phi_{2}\right\rangle-\left\langle\phi_{1}\right\rangle\left\langle\phi_{2}\right\rangle=4 \pi^{2}\left(\left\langle N^{2}\right\rangle-\langle N\rangle^{2}\right)>0,
$$

which means that variables $\phi_{1}$ and $\phi_{2}$ are correlated. On the other hand, if we introduce

$$
\psi_{1,2}=\mathrm{e}^{i \phi_{1,2}}
$$

then

$$
\left\langle\psi_{1,2}\right\rangle=0
$$

and

$$
\begin{aligned}
& \left\langle\psi_{1} \psi_{2}\right\rangle=0 \\
& \left\langle\psi_{1} \psi_{2}\right\rangle-\left\langle\psi_{1}\right\rangle\left\langle\psi_{2}\right\rangle=0,
\end{aligned}
$$

which means that variables $\psi_{1}$ and $\psi_{2}$ are statistically independent. In this illustrative example it is clear that the difference in statistical properties between $\phi$ and $\psi$ arises from the fact that function $\psi(\phi)$ does not have inverse and, consequently, the information about $N$ contained in $\phi$ is lost in $\psi$.

This illustration, although simple, captures the property that actually happens in reality as we will show below. Let us use the following expression for the phase

$$
\phi_{j}=\mathfrak{s} \ln a_{j} .
$$

Substituting (8) and Taylor-expanding of logarithm in $\epsilon$ one gets

$$
\phi_{j}=\mathfrak{s} \ln \left(a_{j}^{(0)}+\epsilon a_{j}^{(1)}+\epsilon^{2} a_{j}^{(2)}\right)=\phi^{(0)}+\epsilon \phi^{(1)}+\epsilon^{2} \phi^{(2)},
$$

where

$$
\begin{aligned}
\phi^{(0)} & =\Im \ln a_{j}^{(0)}, \\
\phi^{(1)} & =\Im \frac{a_{j}^{(1)}}{a_{j}^{(0)}}, \\
\phi^{(2)} & =\Im\left(-\frac{1}{2}\left(\frac{a_{j}^{(1)}}{a_{j}^{(0)}}\right)^{2}+\frac{a_{j}^{(2)}}{a_{j}^{(0)}}\right) .
\end{aligned}
$$

Now let us perform averaging over the statistics of factors $\psi^{(0)}$. As usual, the surviving terms are those in which all $\psi^{(0)}$ 's cancel out due to their pairwise matchings. This is possible only if the number of $\psi^{(0)}$ 's is equal to the number of $\bar{\psi}^{(0)}$ 's in the products defining these terms. Easy to see that the $\epsilon$ term involves three $\psi^{(0)}$ 's and therefore its average is zero. Therefore,

$$
\left\langle\phi_{j}(T)\right\rangle-\left\langle\phi_{j}^{(0)}\right\rangle=\frac{\epsilon^{2}}{A_{j}^{2}} \Im\left\langle-\frac{1}{2 A_{j}^{2}}\left(a_{j}^{(1)} \bar{a}_{j}^{(0)}\right)^{2}+a_{j}^{(2)} \bar{a}_{j}^{(0)}\right\rangle .
$$


Let us consider

$$
\left\langle\left(a_{j}^{(1)} \bar{a}_{j}^{(0)}\right)^{2}\right\rangle=\left\langle\sum_{m, n, \kappa, v}\left(V_{m n}^{j} a_{m} a_{n} \Delta_{m n}^{j} \delta_{m+n}^{j}+2 \bar{V}_{j n}^{m} a_{m} \bar{a}_{n} \bar{\Delta}_{j n}^{m} \delta_{j+n}^{m}\right)\left(V_{\kappa \nu}^{j} a_{\kappa} a_{\nu} \Delta_{\kappa \nu}^{j} \delta_{\kappa+v}^{j}+2 \bar{V}_{j \nu}^{\kappa} a_{\kappa} \bar{a}_{\nu} \bar{\Delta}_{j n}^{m} \delta_{j+n}^{m}\right) \bar{a}_{j}^{2}\right\rangle .
$$

Here, there are two terms with equal number of $\psi^{(0)}$ 's and $\bar{\psi}^{(0)}$ 's but all couplings of index $j$ to any other index give zero because $V=0$ if one of its wavenumbers is zero. Thus, $\left\langle\left(a_{j}^{(1)} \bar{a}_{j}^{(0)}\right)^{2}\right\rangle=0$. The other term, $\left\langle a_{j}^{(2)} \bar{a}_{j}^{(0)}\right\rangle_{\psi}$ has already been calculated before when evaluating $J_{3}$. We have

$$
\left\langle a_{j}^{(2)} \bar{a}_{j}^{(0)}\right\rangle_{\psi}=4 \sum_{m, n}\left[-\left|V_{m n}^{j}\right|^{2} \bar{E}\left(0, \omega_{m n}^{j}\right) \delta_{m+n}^{j} A_{n}^{2}+\left|V_{j n}^{m}\right|^{2} E\left(0,-\omega_{j n}^{m}\right) \delta_{j+n}^{m}\left(A_{m}^{2}-A_{n}^{2}\right)\right] A_{j}^{2} .
$$

Let us take limits $N \rightarrow \infty$ and $T \rightarrow \infty$ and replace $\langle\phi(T)\rangle_{\psi}-\langle\phi(0)\rangle_{\psi} / T$ by $\langle\dot{\phi}\rangle_{\psi}$. We get

$$
\left\langle\dot{\phi}_{j}\right\rangle_{\psi}=\omega_{N L},
$$

where $\omega_{N L}$ is the nonlinear frequency correction given by

$$
\omega_{N L}=4 \epsilon^{2} \int\left[\left|V_{m n}^{j}\right|^{2} \mathcal{P}\left(\frac{1}{\omega_{m n}^{j}}\right) \delta_{m+n}^{j}-\left|V_{j n}^{m}\right|^{2} \mathcal{P}\left(\frac{1}{\omega_{j n}^{m}}\right) \delta_{j+n}^{m}\left(A_{m}^{2}-A_{n}^{2}\right)\right] A_{j}^{2} \mathrm{~d} k_{m} \mathrm{~d} k_{n} .
$$

Here $\mathcal{P}(x)$ denotes the principal value of the integral. Averaging over the amplitudes, we have

$$
\left\langle\dot{\phi}_{j}\right\rangle=\left\langle\omega_{N L}\right\rangle
$$

where $\left\langle\omega_{N L}\right\rangle$ is the amplitude-averaged nonlinear frequency correction

$$
\left\langle\omega_{N L}\right\rangle=4 \epsilon^{2} \int\left[\left|V_{m n}^{j}\right|^{2} \mathcal{P}\left(\frac{1}{\omega_{m n}^{j}}\right) \delta_{m+n}^{j}-\left|V_{j n}^{m}\right|^{2} \mathcal{P}\left(\frac{1}{\omega_{j n}^{m}}\right) \delta_{j+n}^{m}\left(n_{m}-n_{n}\right)\right] n_{j} \mathrm{~d} k_{m} \mathrm{~d} k_{n} .
$$

We can see that the mean value of the phase is steadily changing over the nonlinear time and, therefore, it would be incorrect to assume that the phase "remains uniformly distributed from $-\pi$ to $\pi$ " even though this could be true for $t=0$. This is one of the reasons why we formulate RPA in terms of $\psi$ and not $\phi$. Indeed, $\psi$ was shown above to stay uniformly distributed on the unit circle over the nonlinear time.

The other reason is that, strictly speaking, $\phi$ 's do not stay de-correlated where as $\psi$ 's do (as shown before). We already saw in the beginning of this section that this situation is possible due to the fact that the map $\phi \rightarrow \psi=\mathrm{e}^{i \phi}$ is not a bijection. Let us now study such a build-up in statistical dependence of the phases, let us consider correlator $\mathcal{F}_{j, k} \equiv\left\langle\left(\phi_{j}-\left\langle\phi_{j}\right\rangle\right)\left(\phi_{k}-\left\langle\phi_{k}\right\rangle\right)\right\rangle=\left\langle\phi_{j} \phi_{k}\right\rangle-\left\langle\phi_{j}\right\rangle\left\langle\phi_{k}\right\rangle$. At time $T$ we have

$$
\mathcal{F}_{j, k}(T)=\mathcal{F}_{j, k}^{(0)}+\epsilon \mathcal{F}_{j, k}^{(1)}+\epsilon^{2} \mathcal{F}_{j, k}^{(2)},
$$

where

$$
\begin{aligned}
\mathcal{F}_{j, k}^{(0)} & =\left\langle\phi_{j}^{(0)} \phi_{k}^{(0)}\right\rangle-\left\langle\phi_{j}^{(0)}\right\rangle\left\langle\phi_{k}^{(0)}\right\rangle, \\
\mathcal{F}_{j, k}^{(1)} & =\left\langle\phi_{j}^{(1)} \phi_{k}^{(0)}\right\rangle+\left\langle\phi_{k}^{(1)} \phi_{j}^{(0)}\right\rangle, \\
\mathcal{F}_{j, k}^{(2)} & =\left\langle\phi_{j}^{(1)} \phi_{k}^{(1)}\right\rangle+\left\langle\phi_{j}^{(2)} \phi_{k}^{(0)}\right\rangle+\left\langle\phi_{k}^{(2)} \phi_{j}^{(0)}\right\rangle-\left\langle\phi_{j}^{(2)}\right\rangle\left\langle\phi_{k}^{(0)}\right\rangle-\left\langle\phi_{k}^{(2)}\right\rangle\left\langle\phi_{j}^{(0)}\right\rangle .
\end{aligned}
$$


Here, we have taken into account that, as we showed earlier, $\left\langle\phi_{j}^{(1)}\right\rangle=0$. Let us consider the $\epsilon$-term $\mathcal{F}_{j, k}^{(1)}$, e.g.,

$$
\left\langle\phi_{j}^{(1)} \phi_{k}^{(0)}\right\rangle=\Im\left\langle\left(a_{j}^{(1)} \bar{a}_{j}^{(0)}\right) \phi_{k}^{(0)}\right\rangle=\Im \sum_{k, m, n} V_{m n}^{j}\left\langle a_{m} a_{n} \bar{a}_{j} \phi_{k}\right\rangle \Delta_{m n}^{j} \delta_{m+n}^{j}+2 \bar{V}_{j n}^{m}\left\langle a_{m} \bar{a}_{n} \bar{a}_{j} \phi_{k}\right\rangle \bar{\Delta}_{j n}^{m} \delta_{j+n}^{m} .
$$

In this expression, we have a factor $\phi_{k}$ which enters directly and not via the combination $\psi_{k}=\mathrm{e}^{i \phi_{k}}$. Potentially, this could greatly complicate the situation because to objects like $\left\langle\psi_{k} \phi_{k}\right\rangle$ knowledge of the statistics of $\psi$ is not sufficient and one needs the full PDF of $\phi_{k}$. Fortunately, however, this does not cause problems here because, no matter what index is matched to $k$, matching of the two remaining indices results in $V=0$. Therefore, the contribution of the $\epsilon$ terms is nil.

Let us now consider the $\mathcal{F}_{j, k}^{(2)}$ starting with

$$
\left\langle\phi_{j}^{(2)} \phi_{k}^{(0)}\right\rangle_{\psi}=\left\langle\Im\left[-\frac{1}{2}\left(\frac{a_{j}^{(1)}}{a_{j}^{(0)}}\right)^{2}+\frac{a_{j}^{(2)}}{a_{j}^{(0)}}\right] \phi_{k}^{(0)}\right\rangle=\Im\left\langle\left[-\frac{1}{2 A_{j}^{4}}\left(a_{j}^{(1)} \bar{a}_{j}^{(0)}\right)^{2}+\frac{1}{A_{j}^{2}} a_{j}^{(2)} \bar{a}_{j}^{(0)}\right] \phi_{k}^{(0)}\right\rangle .
$$

We see that the square bracket on the RHS involves an even number (four or six) of $\psi$ 's in each term. Thus, in order for these terms to survive these $\psi$ 's must cancel out which is possible when their indices match in a pairwise way. But this means that index $k$ (of $\phi_{k}$ ) does not match to any of the indices of $\psi$ 's and, therefore, the averaging of $\phi_{k}$ can be taken separately because it is statistically independent of all other phase factors. ${ }^{5}$ Thus, we conclude that $\left\langle\phi_{j}^{(2)} \phi_{k}^{(0)}\right\rangle_{\psi}=\left\langle\phi_{j}^{(2)}\right\rangle_{\psi}\left\langle\phi_{k}^{(0)}\right\rangle_{\psi}$ and these terms drop out of $\mathcal{F}_{j, k}^{(2)}$. The remaining term in $\mathcal{F}_{j, k}^{(2)}$ is

$$
\begin{aligned}
\left\langle\phi_{j}^{(1)} \phi_{k}^{(1)}\right\rangle_{\psi}= & -\frac{1}{4}\left\langle\left(\frac{a_{j}^{(1)}}{a_{j}^{(0)}}-\frac{\bar{a}_{j}^{(1)}}{\bar{a}_{j}^{(0)}}\right)\left(\frac{a_{j}^{(1)}}{a_{j}^{(0)}}-\frac{\bar{a}_{j}^{(1)}}{\bar{a}_{j}^{(0)}}\right)\right\rangle=-\frac{1}{4}\left\langle\frac{a_{j}^{(1)} a_{k}^{(1)}}{a_{j}^{(0)} a_{k}^{(0)}}-\frac{a_{j}^{(1)} \bar{a}_{k}^{(1)}}{a_{j}^{(0)} a_{k}^{(0)}}+c c\right\rangle \\
= & 2\left|V_{k(j-k)}^{j}\right|^{2}\left|\Delta_{k(j-k)}^{j}\right|^{2} A_{j-k}^{2}+2\left|V_{j(k-j)}^{k}\right|^{2}\left|\Delta_{j(k-j)}^{k}\right|^{2} A_{k-j}^{2}+2\left|V_{j k}^{j+k}\right|^{2}\left|\Delta_{j k}^{j+k}\right|^{2} A_{j+k}^{2} \\
& +\frac{\delta_{k}^{j}}{A_{j}^{2}} \sum_{l, m}\left[\left|V_{l m}^{j}\right|^{2}\left|\Delta_{l m}^{j}\right|^{2} \delta_{l+m}^{j}+2\left|V_{j l}^{m}\right|^{2}\left|\Delta_{j l}^{m}\right|^{2} \delta_{l+j}^{m}\right] A_{l}^{2} A_{m}^{2} .
\end{aligned}
$$

We can now average over the amplitudes and take limits $N \rightarrow \infty$ and $\epsilon \rightarrow 0$ and write

$$
\begin{aligned}
\dot{\mathcal{F}}_{j, k}= & 4 \pi \epsilon^{2}\left[\left|V_{k(j-k)}^{j}\right|^{2} \delta\left(\omega_{k(j-k)}^{j}\right) n_{j-k}+\left|V_{j(k-j)}^{k}\right|^{2} \delta\left(\omega_{j(k-j)}^{k}\right) n_{k-j}+\left|V_{j k}^{j+k}\right|^{2} \delta\left(\omega_{j k}^{j+k}\right) n_{j+k}\right] \\
& +\frac{2 \pi \epsilon^{2} \delta_{k}^{j}}{n_{j}} \int\left[\left|V_{l m}^{j}\right|^{2} \delta\left(\omega_{l m}^{j}\right) \delta_{l+m}^{j}+2\left|V_{j l}^{m}\right|^{2} \delta\left(\omega_{j l}^{m}\right) \delta_{l+j}^{m}\right] n_{l} n_{m} \mathrm{~d} k_{l} \mathrm{~d} k_{m} .
\end{aligned}
$$

Presence of the first term on the RHS indicates that the phases of the $j$-th and the $k$-th modes get correlated on the nonlinear time. This correlation is week in a sense that $\mathcal{F}_{j, k}$ has a sharp peak at $j=k$ but the integrated contribution of all $j \neq k$ is of the same order as the value at the contribution of the $j=k$ peak and, therefore, could cause a problem should one tried to build RPA based on the statistics of $\phi$ 's rather than $\psi$ 's (which remain de-correlated).

Let us consider a special case of (61) for $j=k$ which is interesting because it allows one to calculate the dispersion in phases,

$$
\sigma_{k}=\left\langle\phi_{k}^{2}\right\rangle-\left\langle\phi_{k}\right\rangle^{2}
$$

\footnotetext{
5 There is of course also a possibility that $k$ couples simultaneously to both indices in a pair, but this contribution contains $\sim 1 / N$ less terms and, therefore, should be ignored.
} 
We have

$$
\dot{\sigma}_{k}=\frac{\eta_{k}}{n_{k}}
$$

where $\eta_{k}$ is defined in (44) and $n_{k}=\left\langle\left|a_{k}\right|^{2}\right\rangle$. One can see that the RHS here is always positive and, therefore, the phase fluctuations experience an unlimited growth. On stationary spectra, this growth is $\sim \sqrt{t}$ which corresponds to $\sigma \sim t$. Recall that the mean value of the phase is also changing in time with the rate $\omega_{N L}$ and on stationary spectra this change is linear in time.

\section{Discussion}

In the present paper, we considered evolution of the full $\mathrm{N}$-mode objects such as the generating functional and the probability density function for all the wave amplitudes and their phase factors. We proved that the phase factors, being statistically independent and uniform on $S^{1}$ initially, remain so over the nonlinear evolution time in the leading order in small nonlinearity. If in addition the initial amplitudes are independent too, then they remain so over the nonlinear time in a weak sense. Namely, all joint PDF's for the number of modes $M \ll N$ split into products of the one-mode densities with $\mathrm{O}(M / N)$ and $\mathrm{O}\left(\epsilon^{2}\right)$ accuracy. Thus, the full $N$-mode PDF does not factorise as a product of $N$ one-mode densities and the Fourier modes in the set considered as a whole are not independent. However, the wave turbulence closure only deals with the joint objects of the finite size $M$ of variables while taking $N \rightarrow \infty$ limit. These objects do factorise into products and, for the WT purposes, the Fourier modes can be interpreted as statistically independent. In particular, the derivation of the kinetic equation for the energy spectrum deals only with the one-mode and the two-mode distributions and is, therefore, justified by the results of the present paper. Generally speaking, our results reduce the leading-order WT problem to the study of the one-mode amplitude PDF's and they validate the generalised RPA technique introduced in $[19,20]$. Such a study of the one-mode PDF and the high-order momenta of the wave amplitudes was done in $[19,20]$. It was shown, in particular, that anomalous probabilities of large wave amplitudes can appear in the form of a finite-flux solution in the amplitude space caused by a wave-breaking amplitude cutoff. The reader is referred to these papers for the discussion of the WT intermittency.

Although our results indicate that correlations between 2 or more (but $\ll N$ ) modes do not appear in the leading (i.e., $\epsilon^{2}$ ) order for the three-wave systems, they definitely appear as corrections in the next (i.e., $\epsilon^{4}$ ) order. Our paper is concerned with the main order statistics only in which the main evolution happens in the one-mode objects, e.g., the one-mode amplitude distributions. For study of the multi-mode correlations developing in WT in the next order in $\epsilon$ the reader is referred to papers [21,22].

We have also considered correlators of the phase and we showed the relation between the statistical properties of the phase $\phi$ and the phase factors $\psi=\mathrm{e}^{i \phi}$. We showed that the mean of $\phi$ and its fluctuations about the mean grow in time and, therefore, there exist no $2 \pi$-wide interval in which the phase would remain uniformly distributed. Moreover, phases $\phi$ become correlated at different wavenumbers that lie on the resonant manifold. These properties make the phase $\phi$ an inappropriate variable for formulating the RPA method of WT description. On the other hand, our work shows that the phase factors $\psi=\mathrm{e}^{i \phi}$ do remain statistically independent and uniform on $S^{1}$ which makes them the right choice for the RPA formulation.

The present paper deals with the three-wave systems only. The four-wave resonant interactions are slightly more complicated in that the nonlinear frequency shift occurs at a lower order in nonlinearity parameter than the nonlinear evolution of the wave amplitudes. To build a consistent description of the amplitude moments one has to perform a renormalisation of the perturbation series taking into account the nonlinear frequency shift. This derivation will be published separately, whereas here we just announce its main result, the four-wave generalisation of the Peierls 
equation for the PDF. It has the same continuity equation form (36) but now the probability flux is

$$
F_{j}=4 \pi \epsilon^{4} \sum_{123} W_{23}^{j 1} \delta\left(\tilde{\omega}_{23}^{j i}\right) \delta_{23}^{j 1} s_{1} s_{2} s_{3} s_{j}\left(\frac{\delta}{\delta s_{j}}+\frac{\delta}{\delta s_{1}}-\frac{\delta}{\delta s_{2}}-\frac{\delta}{\delta s_{3}}\right) P,
$$

where $W_{23}^{j 1}$ is the four-wave interaction coefficient and $\tilde{\omega}_{\mu \nu}^{l \alpha}=\omega_{\mu \nu}^{l \alpha}+\Omega_{l}+\Omega_{\alpha}-\Omega_{\mu}-\Omega_{v}$ with $\Omega_{l}=$ $2 \epsilon \sum_{\mu} W_{l \mu}^{l \mu} n_{\mu}$ being the nonlinear frequency shift. As wee see this equation is even more compact than its threewave analog. In addition to the derivation of this equation, we will also analyse its properties and consequences for the mode correlations and intermittency in four-wave turbulent systems.

\section{Acknowledgements}

Yeontaek Choi's work is supported by KOSEF M07-2003-000-10003-0. Yuri Lvov acknowledges support provided by NSF CAREER grant DMS 0134955 and by ONR YIP grant N000140210528.

\section{Appendix A}

Let us obtain $Z(T)$ in terms of the series in small nonlinearity up to the second order in $\epsilon$. As an intermediate step, we first consider separately the amplitude and the phase ingredients of $Z$ and substitute the $\epsilon$-expansion of $a$ from (8) into their expressions,

$$
\begin{aligned}
& \mathrm{e}^{\lambda_{j}\left|a_{j}\right|^{2}}=\mathrm{e}^{\lambda_{j}\left|a_{j}^{(0)}+\epsilon a_{j}^{(1)}+\epsilon^{2} a_{j}^{(2)}\right|^{2}}=\mathrm{e}^{\lambda_{j}\left|a_{j}^{(0)}\right|^{2}+\epsilon \lambda_{j}\left(a_{j}^{(1)} \bar{a}_{j}^{(0)}+\bar{a}_{j}^{(1)} a_{j}^{(0)}\right)+\epsilon^{2} \lambda_{j}\left[\left|a_{j}^{(1)}\right|^{2}+\left(a_{j}^{(2)} \bar{a}_{j}^{(0)}+\bar{a}_{j}^{(2)} a_{j}^{(0)}\right)\right]} \\
& =\mathrm{e}^{\lambda_{j}\left|a_{j}^{(0)}\right|^{2}}\left\{1+\epsilon \lambda_{j}\left(a_{j}^{(1)} \bar{a}_{j}^{(0)}+\bar{a}_{j}^{(1)} a_{j}^{(0)}\right)+\epsilon^{2} \lambda_{j}\left[\left|a_{j}^{(1)}\right|^{2}+\left(a_{j}^{(2)} \bar{a}_{j}^{(0)}+\bar{a}_{j}^{(2)} a_{j}^{(0)}\right)\right]+\frac{\epsilon^{2} \lambda_{j}^{2}}{2}\left(a_{j}^{(1)} \bar{a}_{j}^{(0)}+\bar{a}_{j}^{(1)} a_{j}^{(0)}\right)^{2}\right\} \\
& =\mathrm{e}^{\lambda_{j} A_{j}^{(0) 2}}\left(1+\epsilon \alpha_{1 j}+\epsilon^{2} \alpha_{2 j}\right),
\end{aligned}
$$

and

$$
\begin{gathered}
\psi_{j}^{\mu_{j}}=\left(\frac{a_{j}^{(0)}+\epsilon a_{j}^{(1)}+\epsilon^{2} a_{j}^{(2)}}{\bar{a}_{j}^{(0)}+\epsilon \bar{a}_{j}^{(1)}+\epsilon^{2} \bar{a}_{j}^{(2)}}\right)^{\frac{\mu_{j}}{2}}=\psi_{j}^{(0) \mu_{j}}\left(\frac{1+\epsilon\left(a_{j}^{(1)} / a_{j}^{(0)}\right)+\epsilon^{2}\left(a_{j}^{(2)} / a_{j}^{(0)}\right)}{1+\epsilon\left(\bar{a}_{j}^{(1)} / \bar{a}_{j}^{(0)}\right)+\epsilon^{2}\left(\bar{a}_{j}^{(2)} / \bar{a}_{j}^{(0)}\right)}\right)^{\frac{\mu_{j}}{2}} \\
=\psi_{j}^{(0) \mu_{j}}\left[1+\epsilon \frac{\mu_{j}}{2} \frac{a_{j}^{(1)}}{a_{j}^{(0)}}+\epsilon^{2} \frac{\mu_{j}}{2} \frac{a_{j}^{(2)}}{a_{j}^{(0)}}+\frac{\epsilon^{2}}{2} \frac{\mu_{j}}{2}\left(\frac{\mu_{j}}{2}-1\right)\left(\frac{a_{j}^{(1)}}{a_{j}^{(0)}}\right)^{2}\right] \\
\times\left[1-\epsilon \frac{\mu_{j}}{2} \frac{a_{j}^{(1)}}{a_{j}^{(0)}}-\epsilon^{2} \frac{\mu_{j}}{2} \frac{a_{j}^{(2)}}{a_{j}^{(0)}}+\frac{\epsilon^{2}}{2} \frac{\mu_{j}}{2}\left(\frac{\mu_{j}}{2}+1\right)\left(\frac{a_{j}^{(1)}}{a_{j}^{(0)}}\right)^{2}\right] \\
=\psi_{j}^{(0) \mu_{j}}\left\{1+\epsilon \frac{\mu_{j}}{2}\left(\frac{a_{j}^{(1)}}{a_{j}^{(0)}}-\frac{\bar{a}_{j}^{(1)}}{\bar{a}_{j}^{(0)}}\right)+\epsilon^{2}\left[\frac{\mu_{j}}{2}\left(\frac{a_{j}^{(2)}}{a_{j}^{(0)}}-\frac{\bar{a}_{j}^{(2)}}{\bar{a}_{j}^{(0)}}\right)+\frac{\mu_{j}}{4}\left[\left(\frac{\mu_{j}}{2}-1\right)\left(\frac{a_{j}^{(1)}}{a_{j}^{(0)}}\right)^{2}\right.\right.\right. \\
\left.\left.\left.+\left(\frac{\mu_{j}}{2}+1\right)\left(\frac{a_{j}^{(1)}}{a_{j}^{(0)}}\right)^{2}\right]-\frac{\mu_{j}^{2}\left|a_{j}^{(1)}\right|^{2}}{4 A_{j}^{(0) 2}}\right]\right\}=\psi_{j}^{(0) \mu_{j}}\left(1+\epsilon \beta_{1 j}+\epsilon^{2} \beta_{2 j}\right),
\end{gathered}
$$


where $\alpha_{1 j}, \alpha_{2 j}, \beta_{1 j}$ and $\beta_{2 j}$ denote the linear and quadratic contributions into the amplitude and phase parts of $Z$ respectively,

$$
\begin{aligned}
& \alpha_{1 j}=\lambda_{j}\left(a_{j}^{(1)} \bar{a}_{j}^{(0)}+\bar{a}_{j}^{(1)} a_{j}^{(0)}\right) \\
& \alpha_{2 j}=\lambda_{j}+\lambda_{j}^{2} A_{j}^{(0) 2}\left|a_{j}^{(1)}\right|^{2}+\lambda_{j}\left(a_{j}^{(2)} \bar{a}_{j}^{(0)}+\bar{a}_{j}^{(2)} a_{j}^{(0)}\right)+\frac{\lambda_{j}^{2}}{2}\left(a_{j}^{(1)} \bar{a}_{j}^{(0)}\right)^{2}+\left(\bar{a}_{j}^{(1)} a_{j}^{(0)}\right)^{2}, \\
& \beta_{1 j}=\frac{\mu_{j}}{2 A_{j}^{(0) 2}}\left(a_{j}^{(1)} \bar{a}_{j}^{(0)}-\bar{a}_{j}^{(1)} a_{j}^{(0)}\right), \\
& \beta_{2 j}=\frac{\mu_{j}}{2 A_{j}^{(0) 2}}\left(a_{j}^{(2)} \bar{a}_{j}^{(0)}-\bar{a}_{j}^{(2)} a_{j}^{(0)}\right)+\frac{\mu_{j}}{4}\left[\left(\frac{\mu_{j}}{2}-1\right)\left(\frac{a_{j}^{(1)}}{a_{j}^{(0)}}\right)^{2}+\left(\frac{\mu_{j}}{2}+1\right)\left(\frac{\bar{a}_{j}^{(1)}}{\bar{a}_{j}^{(0)}}\right)^{2}\right]-\frac{\mu_{j}^{2}\left|a_{j}^{(1)}\right|^{2}}{4 A_{j}^{(0) 2}} .
\end{aligned}
$$

Substituting expansions (64) and (65) into the expression for $Z$, we have

$$
\begin{aligned}
& Z(T)=\frac{1}{(2 \pi)^{N}}\left\langle\prod_{l} \mathrm{e}^{\lambda_{l}\left|a_{l}\right|^{2}}\left(\frac{a_{l}}{\bar{a}_{l}}\right)^{\frac{\mu_{l}}{2}}\right\rangle=\frac{1}{(2 \pi)^{N}}\left\langle\prod_{l} \mathrm{e}^{\lambda_{l} A_{l}^{(0) 2}}\left[1+\epsilon \alpha_{1 l}+\epsilon^{2} \alpha_{2 l}\right] \psi_{l}^{(0) \mu_{l}}\left[1+\epsilon \beta_{1 l}+\epsilon^{2} \beta_{2 l}\right]\right\rangle \\
& =\frac{1}{(2 \pi)^{N}}\left\langle\prod _ { l } \mathrm { e } ^ { \lambda _ { l } A _ { l } ^ { ( 0 ) 2 } } \psi _ { l } ^ { ( 0 ) \mu _ { l } } \left[ 1+\epsilon \sum_{j}\left(\alpha_{1 j}+\beta_{1 j}\right)+\epsilon^{2} \sum_{j}\left(\alpha_{2 j}+\beta_{2 j}\right)\right.\right. \\
& \left.\left.+\epsilon^{2} \sum_{j<k}\left(\alpha_{1 j} \alpha_{1 k}+\beta_{1 j} \beta_{1 k}\right)+\sum_{j, k} \alpha_{1 j} \beta_{1 k}\right]\right\rangle \\
& =\frac{1}{(2 \pi)^{N}}\left\langle\prod_{l} \mathrm{e}^{\lambda_{l} A_{l}^{(0) 2}} \psi_{l}^{(0) \mu_{l}}[1+\underbrace{\epsilon \sum_{j}\left(\alpha_{1 j}+\beta_{1 j}\right)}_{I_{1}}+\underbrace{\sum_{j}\left(\alpha_{2 j}+\beta_{2 j}+\alpha_{1 j} \beta_{1 j}\right)}_{I_{2}}\right. \\
& +\epsilon^{2} \underbrace{\frac{1}{2} \sum_{j \neq k}\left(\alpha_{1 j} \alpha_{1 k}+\beta_{1 j} \beta_{1 k}+2 \alpha_{1 j} \beta_{1 k}\right)}_{I_{3}}]\rangle \text {. }
\end{aligned}
$$

For parts $I_{1}, I_{2}$ and $I_{3}$ in the above expression we have,

$$
\begin{aligned}
& I_{1}=\sum_{j}\left(\lambda_{j}+\frac{\mu_{j}}{2 A_{j}^{2}}\right) a_{j}^{(1)} \bar{a}_{j}^{(0)}+\left(\lambda_{j}-\frac{\mu_{j}}{2 A_{j}^{2}}\right) \bar{a}_{j}^{(1)} a_{j}^{(0)} \\
& I_{2}=\sum_{j}\left(\lambda_{j}+\lambda_{j}^{2} A_{j}^{2}-\frac{\mu_{j}^{2}}{2 A_{j}^{2}}\right)\left|a_{j}^{(1)}\right|^{2}+\left(\lambda_{j}+\frac{\mu_{j}}{2 A_{j}^{2}}\right) a_{j}^{(2)} \bar{a}_{j}^{(0)}+\left(\lambda_{j}-\frac{\mu_{j}}{2 A_{j}^{2}}\right) \bar{a}_{j}^{(2)} a_{j}^{(0)}
\end{aligned}
$$




$$
\begin{gathered}
+\left[\frac{\lambda_{j}^{2}}{2}+\frac{\mu_{j}}{4 A_{j}^{4}}\left(\frac{\mu_{j}}{2}-1\right)+\frac{\lambda_{j} \mu_{j}}{2 A_{j}^{2}}\right]\left(a_{j}^{(1)} \bar{a}_{j}^{(0)}\right)^{2}+\left[\frac{\lambda_{j}^{2}}{2}+\frac{\mu_{j}}{4 A_{j}^{4}}\left(\frac{\mu_{j}}{2}+1\right)-\frac{\lambda_{j} \mu_{j}}{2 A_{j}^{2}}\right]\left(\bar{a}_{j}^{(1)} a_{j}^{(0)}\right)^{2}, \\
I_{3}=\frac{1}{2} \sum_{j \neq k}\left[\lambda_{j} \lambda_{k}\left(a_{j}^{(1)} \bar{a}_{j}^{(0)}+\bar{a}_{j}^{(1)} a_{j}^{(0)}\right)\left(a_{k}^{(1)} \bar{a}_{k}^{(0)}+\bar{a}_{k}^{(1)} a_{k}^{(0)}\right)+\frac{\lambda_{j} \mu_{k}}{A_{k}^{2}}\left(a_{j}^{(1)} \bar{a}_{j}^{(0)}+\bar{a}_{j}^{(1)} a_{j}^{(0)}\right)\left(a_{k}^{(1)} \bar{a}_{k}^{(0)}-\bar{a}_{k}^{(1)} a_{k}^{(0)}\right)\right. \\
\left.+\frac{\mu_{j} \mu_{k}}{4 A_{j}^{2} A_{k}^{2}}\left(a_{j}^{(1)} \bar{a}_{j}^{(0)}-\bar{a}_{j}^{(1)} a_{j}^{(0)}\right)\left(a_{k}^{(1)} \bar{a}_{k}^{(0)}-\bar{a}_{k}^{(1)} a_{k}^{(0)}\right)\right],
\end{gathered}
$$

Exploiting the property $\bar{Z}\{\lambda,-\mu\}=Z\{\lambda, \mu\}$ we can write

$$
Z\{\lambda, \mu\}=X\{\lambda, \mu\}+\bar{X}\{\lambda,-\mu\} .
$$

At $t=T$ we have for $X\{\lambda, \mu\}$

$$
X(T)=X(0)+(2 \pi)^{2 N}\left\langle\prod_{\|l\|<N} \mathrm{e}^{\lambda_{l}\left|a_{l}^{(0)}\right|^{2}}\left[\epsilon J_{1}+\epsilon^{2}\left(J_{2}+J_{3}+J_{4}+J_{5}\right)\right]\right\rangle_{A},
$$

where

$$
\begin{aligned}
J_{1}= & \left\langle\prod_{l} \psi_{l}^{(0) \mu_{l}} \sum_{j}\left(\lambda_{j}+\frac{\mu_{j}}{2\left|a_{j}^{(0)}\right|^{2}}\right) a_{j}^{(1)} \bar{a}_{j}^{(0)}\right\rangle_{\psi}, \\
J_{2}= & \frac{1}{2}\left\langle\prod_{l} \psi_{l}^{(0) \mu_{l}} \sum_{j}\left(\lambda_{j}+\lambda_{j}^{2}\left|a_{j}^{(0)}\right|^{2}-\frac{\mu_{j}^{2}}{2\left|a_{j}^{(0)}\right|^{2}}\right)\left|a_{j}^{(1)}\right|^{2}\right\rangle_{\psi}, \\
J_{3}= & \left\langle\prod_{l} \psi_{l}^{(0) \mu_{l}} \sum_{j}\left(\lambda_{j}+\frac{\mu_{j}}{2\left|a_{j}^{(0)}\right|^{2}}\right) a_{j}^{(2)} \bar{a}_{j}^{(0)}\right\rangle_{\psi}, \\
J_{4}= & \left\langle\prod_{l} \psi_{l}^{(0) \mu_{l}} \sum_{j}\left[\frac{\lambda_{j}^{2}}{2}+\frac{\mu_{j}}{4\left|a_{j}^{(0)}\right|^{4}}\left(\frac{\mu_{j}}{2}-1\right)+\frac{\lambda_{j} \mu_{j}}{2\left|a_{j}^{(0)}\right|^{2}}\right]\left(a_{j}^{(1)} \bar{a}_{j}^{(0)}\right)^{2}\right\rangle_{\psi}, \\
J_{5}= & \frac{1}{2}\left\langle\prod_{l} \psi_{l}^{(0) \mu_{l}} \sum_{j \neq k} \lambda_{j} \lambda_{k}\left(a_{j}^{(1)} \bar{a}_{j}^{(0)}+\bar{a}_{j}^{(1)} a_{j}^{(0)}\right) a_{k}^{(1)} \bar{a}_{k}^{(0)}\right. \\
& \left.+\left(\lambda_{j}+\frac{\mu_{j}}{4\left|a_{j}^{(0)}\right|^{2}}\right) \frac{\mu_{k}}{\left|a_{k}^{(0)}\right|^{2}}\left(a_{k}^{(1)} \bar{a}_{k}^{(0)}-\bar{a}_{k}^{(1)} a_{k}^{(0)}\right) a_{j}^{(1)} \bar{a}_{j}^{(0)}\right\rangle_{\psi},
\end{aligned}
$$


where $\langle\cdot\rangle_{A}$ and $\langle\cdot\rangle_{\psi}$ denote the averaging over the initial amplitudes and initial phases respectively. We remind that such individual averages are possible because the amplitudes and the phases are statistically independent from each other at $t=0$.

\section{Appendix B}

\section{B.1. Calculation of $\mathrm{J}_{3}$}

$$
\begin{aligned}
J_{3}= & \left\langle\prod_{l} \psi_{l}^{(0) \mu_{l}} \sum_{j}\left(\lambda_{j}+\frac{\mu_{j}}{2 A_{j}^{2}}\right) a_{j}^{(2)} \bar{a}_{j}^{(0)}\right\rangle_{\psi} \\
= & \left\langle\prod _ { l } \psi _ { l } ^ { ( 0 ) \mu _ { l } } \sum _ { j } ( \lambda _ { j } + \frac { \mu _ { j } } { 2 A _ { j } ^ { 2 } } ) \sum _ { j , m , n , \kappa , v } \left[2 V _ { m n } ^ { j } \left(-V_{\kappa \nu}^{m} a_{n} a_{\kappa} a_{\nu} E\left[\omega_{n \kappa \nu}^{j}, \omega_{m n}^{j}\right] \delta_{\kappa+\nu}^{m}\right.\right.\right. \\
& \left.-2 \bar{V}_{m \nu}^{\kappa} a_{n} a_{\kappa} \bar{a}_{\nu} \bar{E}\left[\omega_{n \kappa}^{j \nu}, \omega_{m n}^{j}\right] \delta_{m+v}^{\kappa}\right) \delta_{m+n}^{j}+2 \bar{V}_{j n}^{m}\left(-V_{\kappa \nu}^{m} \bar{a}_{n} a_{\kappa} a_{\nu} E\left[\omega_{\kappa \nu}^{j n},-\omega_{j n}^{m}\right] \delta_{\kappa+\nu}^{m}\right. \\
& \left.-2 \bar{V}_{m \nu}^{\kappa} \bar{a}_{n} a_{\kappa} \bar{a}_{\nu} E\left[-\omega_{n v j}^{\kappa},-\omega_{j n}^{m}\right] \delta_{m+\nu}^{\kappa}\right) \delta_{j+n}^{m}+2 \bar{V}_{j n}^{m}\left(\bar{V}_{\kappa \nu}^{n} a_{m} \bar{a}_{\kappa} \bar{a}_{\nu} \delta_{\kappa+\nu}^{n} E\left[-\omega_{j v \kappa}^{m},-\omega_{j n}^{m}\right]\right. \\
& \left.\left.\left.+2 V_{n v}^{\kappa} a_{m} \bar{a}_{\kappa} a_{v} E\left[\omega_{v m}^{\kappa j},-\omega_{j n}^{m}\right] \delta_{n+v}^{\kappa}\right) \delta_{j+n}^{m}\right] \bar{a}_{j}\right\rangle_{\psi} .
\end{aligned}
$$

The terms to be averaged here can be drawn as
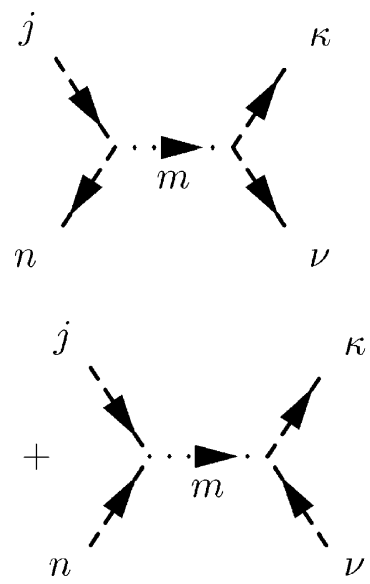
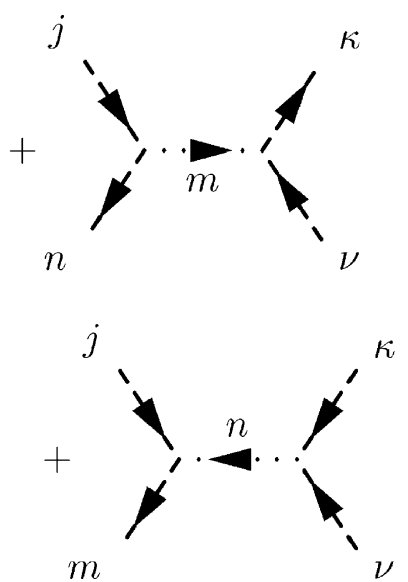
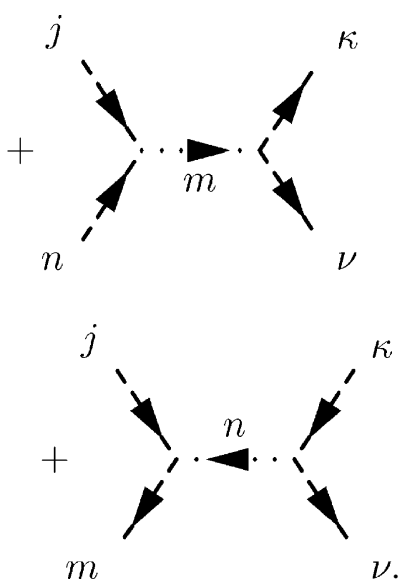

Let us now average over the random phases. Again, the leading order terms will be given by the diagrams with the largest number of internal couplings. They will arise from the $V \bar{V}$ terms (the 2nd, 3rd and the 6th graphs) because they allow 2 internal couplings in each of them. There are also possibilities to have one internal and two external couplings of the dashed lines-such terms will give a $\mathrm{O}(1 / N)$ correction to the leading order. Therefore,
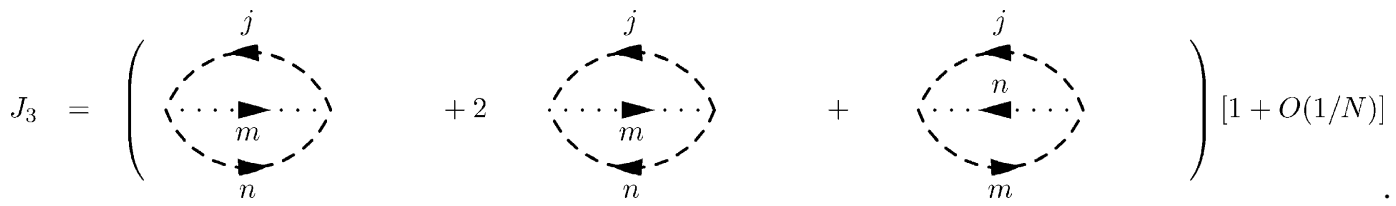


\section{B.2. Calculation of $J_{4}$}

$$
\begin{aligned}
J_{4}= & \left\langle\prod_{l} \psi_{l}^{(0) \mu_{l}} \sum_{j}\left[\frac{\lambda_{j}^{2}}{2}+\frac{\mu_{j}}{4 A_{j}^{4}}\left(\frac{\mu_{j}}{2}-1\right)+\frac{\lambda_{j} \mu_{j}}{2 A_{j}^{2}}\right]\left(a_{j}^{(1)} \bar{a}_{j}^{(0)}\right)^{2}\right\rangle_{\psi} \\
= & \left\langle\prod_{l} \psi_{l}^{\mu_{l}} \sum_{j, m, n, \kappa, \nu}\left[\frac{\lambda_{j}^{2}}{2}+\frac{\mu_{j}}{4 A_{j}^{4}}\left(\frac{\mu_{j}}{2}-1\right)+\frac{\lambda_{j} \mu_{j}}{2 A_{j}^{2}}\right]\right. \\
& \left.\times\left(V_{m n}^{j} a_{m} a_{n} \Delta_{m n}^{j} \delta_{m+n}^{j}+2 \bar{V}_{j n}^{m} a_{m} \bar{a}_{n} \bar{\Delta}_{j n}^{m} \delta_{j+n}^{m}\right)\left(V_{\kappa \nu}^{j} a_{\kappa} a_{\nu} \Delta_{\kappa \nu}^{j} \delta_{\kappa+\nu}^{j}+2 \bar{V}_{j v}^{\kappa} a_{\kappa} \bar{a}_{\nu} \bar{\Delta}_{j n}^{m} \delta_{j+n}^{m}\right) \bar{a}_{j}^{2}\right\rangle_{\psi} .
\end{aligned}
$$

Graphically, the 4 terms to be averaged in this expression are
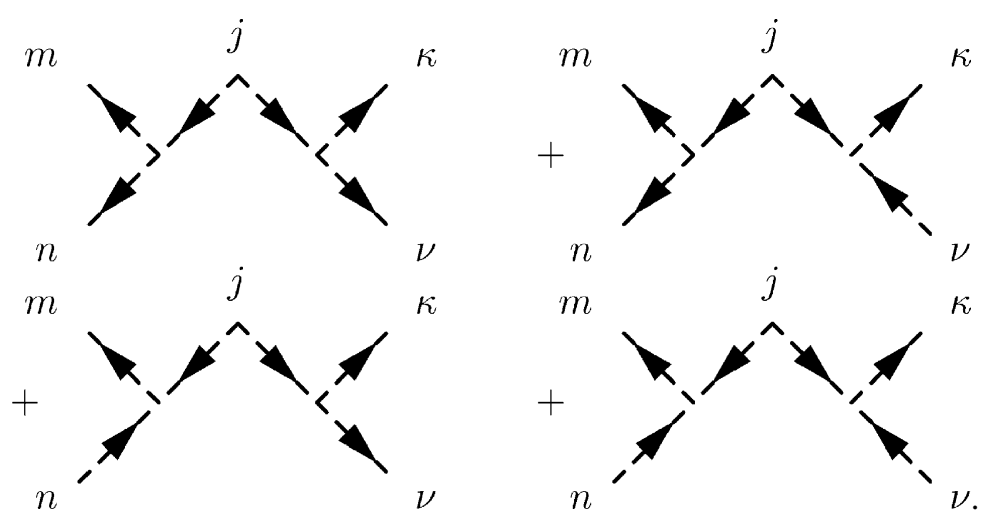

Note that there is no dotted lines in these graphs because for each summation index there is a corresponding wave amplitude present. As a consequence, the rule for the number of surviving summations is somewhat different from what we had so far. Namely, the number of the summation indices after the phase averaging is one less than the number of the purely internal couplings. Easy to see that the phase averaging of the above terms always leads to an external coupling of the dashed lines $j$ which removes the $j$ summation. Moreover, no more than one purely internal coupling of the dashed lines is possible in any of these graphs. ${ }^{6}$ Thus, $J_{4}$ contains no summation at all and is only a $\mathrm{O}\left(1 / N^{2}\right)$ correction to the main terms in $J_{2}$ and $J_{3}$.

\section{B.3. Calculation of $J_{5}$}

Expression for $J_{5}$ seemingly involves a great number terms. However, this number can be dramatically reduced by the following speculation. Just as in $J_{4}$ there is no dotted lines in the graphs involved in $J_{5}$ because for each summation index there is a corresponding wave amplitude present. Thus, we have the same rule for the number of summations surviving the phase averaging (i.e., one less than the number of internal couplings). In order to be of the same order as the leading terms in $J_{2}$ and $J_{3}$, we must have three purely internal couplings and, therefore, no external couplings. This is only possible when the number of dotted lines directed to the vertices is equal to the number of them pointing away which is true for the $V \bar{V}$ terms but not true for the $V V$ and $\bar{V} \bar{V}$ terms. Thus, we

\footnotetext{
${ }^{6}$ The only possibility of the double internal coupling would be in the last graph via joining $m$ with $v$ and $\kappa$ with $n$, but this would mean $j=0$ because of the $\delta$-symbols and, therefore, this term is nil.
} 
will only consider the $V \bar{V}$ terms. Further, the fact that there is no external couplings means that such terms are only non-zero when all $\mu$ 's are zero. Thus, there will be no contribution from the second part of $J_{5}$ which has a $\mu_{k}$ prefactor.

$$
J_{5}=\frac{1}{2}\left\langle\prod_{l} \psi_{l}^{(0) \mu_{l}} \sum_{j \neq k} \lambda_{j} \lambda_{k}\left(a_{j}^{(1)} \bar{a}_{j}^{(0)}+\bar{a}_{j}^{(1)} a_{j}^{(0)}\right) a_{k}^{(1)} \bar{a}_{k}^{(0)}\right\rangle_{\psi}[1+\mathrm{O}(1 / N)],
$$

where the $V \bar{V}$ terms to be averaged here are
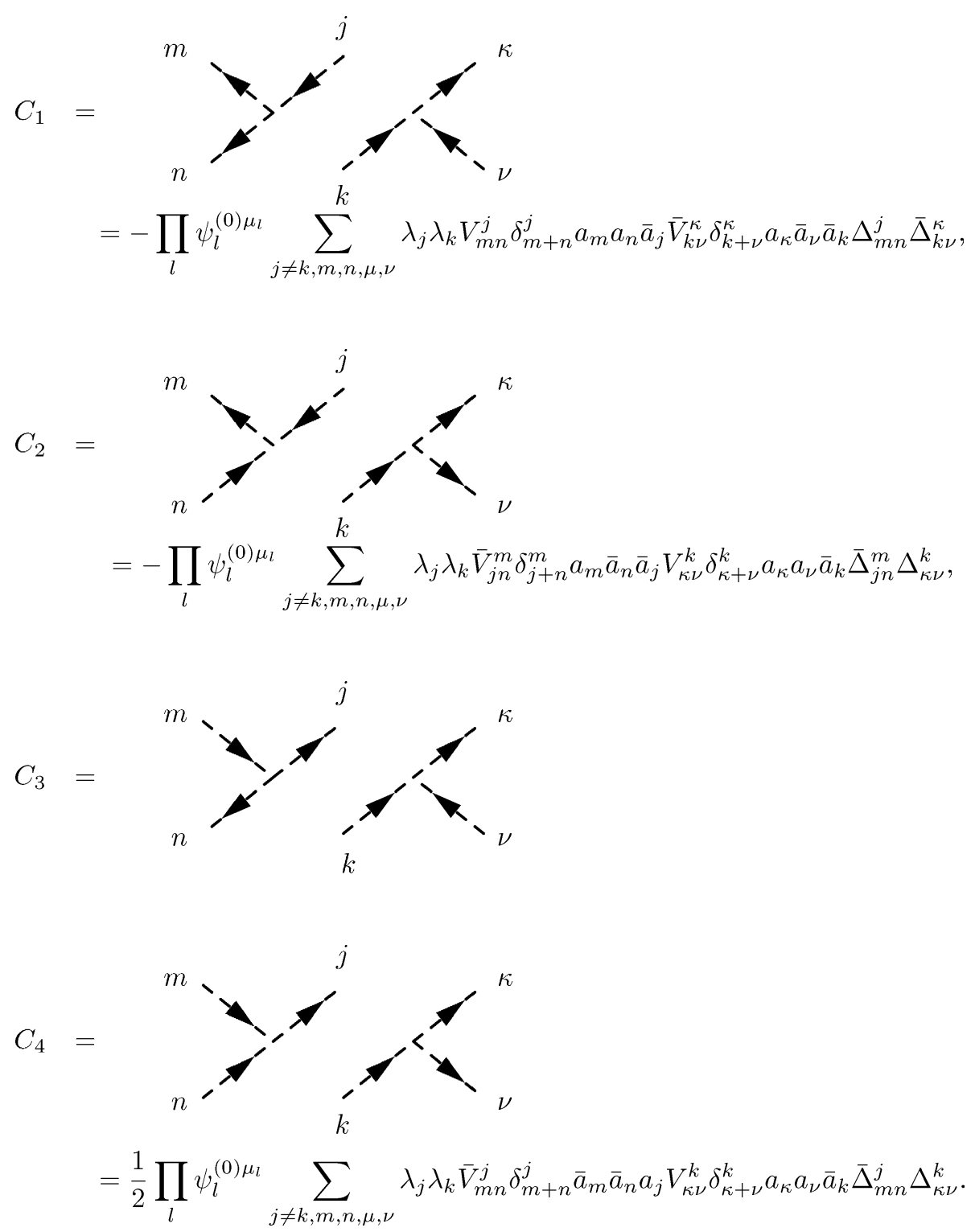
By coupling the dashed lines we have in the leading order

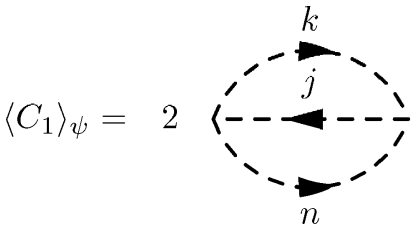<smiles>CC(C)=[W]</smiles>

$\left\langle C_{3}\right\rangle_{\psi}=$

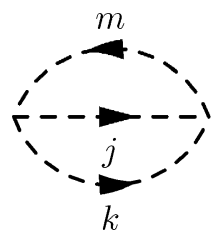

$$
=-2 \prod_{l} \delta\left(\mu_{l}\right) \sum_{j \neq k, n} \lambda_{j} \lambda_{k}\left|V_{k n}^{j}\right|^{2} \delta_{k+n}^{j} A_{j}^{2} A_{n}^{2} A_{k}^{2}\left|\Delta_{k n}^{j}\right|^{2},
$$$$
=-2 \prod_{l} \delta\left(\mu_{l}\right) \sum_{j \neq k, n} \lambda_{j} \lambda_{k}\left|V_{j n}^{k}\right|^{2} \delta_{j+n}^{k} A_{j}^{2} A_{n}^{2} A_{k}^{2}\left|\Delta_{j n}^{k}\right|^{2}=\left\langle C_{1}\right\rangle_{\psi},
$$$$
=2 \prod_{l} \delta\left(\mu_{l}\right) \sum_{j \neq k, m} \lambda_{j} \lambda_{k}\left|V_{j k}^{m}\right|^{2} \delta_{j+k}^{m} A_{j}^{2} A_{m}^{2} A_{k}^{2}\left|\Delta_{j k}^{m}\right|^{2}
$$

Term $C_{4}$ does not survive the averaging because $j \neq k$ and a triple internal coupling is not possible. Summarising,

$$
J_{5}=2 \prod_{l} \delta\left(\mu_{l}\right) \sum_{j \neq k, n} \lambda_{j} \lambda_{k}\left[-2\left|V_{k n}^{j}\right|^{2} \delta_{k+n}^{j}\left|\Delta_{k n}^{j}\right|^{2}+\left|V_{j k}^{n}\right|^{2} \delta_{j+k}^{n}\left|\Delta_{j k}^{n}\right|^{2}\right] A_{j}^{2} A_{n}^{2} A_{k}^{2}[1+\mathrm{O}(1 / N)] \text {. }
$$

\section{References}

[1] V.E. Zakharov, V.S. L'vov, G. Falkovich, Kolmogorov Spectra of Turbulence, Springer-Verlag, 1992.

[2] D.J. Benney, P. Saffman, Proc. R. Soc. A 289 (1966) 301-320;

B.J. Benney, A.C. Newell, Stud. Appl. Math. 48 (1) (1969) 29.

[3] A.A. Galeev, R.Z. Sagdeev, M.A. Leontovich (Eds.), Reviews of Plasma Physics, vol. 6, Consultants Bureau, New York, 1973.

[4] A.C. Newell, Rev. Geophys. 6 (1968) 1.

[5] V.E. Zakharov, Filonenko, J. Appl. Mech. Tech. Phys. 4 (1967) 506-515.

[6] K. Hasselmann, Freely decaying weak turbulence for sea surface gravity waves, J. Fluid Mech. 12 (1962) 481.

[7] S. Dyachenko, A.C. Newell, A. Pushkarev, V.E. Zakharov, Physica D 57 (1992) 96.

[8] H.W. Wyld, Ann. Phys. 14 (1961) 143.

[9] V.S. Lvov, Y.V. Lvov, A.C. Newell, V.E. Zakharov, Statistical description of acoustic turbulence, Phys. Rev. E 56 (1997) 390.

[10] V.E. Zakharov, V.S. Lvov, Izv, Vuzov Radiophys. XVIII (1975) 1470.

[11] R.C. Davidson, Methods in Nonlinear Plasma Theory, Academic Press, New York, 1972.

[12] A.N. Pushkarev, V.E. Zakharov, Turbulence of capillary waves, PRL 76 (1996) 3320-3323; A.N. Pushkarev, V.E. Zakharov, Turbulence of capillary waves, Physica D 135 (2000) 98.

[13] A.M. Balk, S.V. Nazarenko, On the physical realizability of anisotropic kolmogorov spectra of weak turbulence, Sov. Phys. JETP 70 (1990) 1031.

[14] Yu.V. Lvov, E.G. Tabak, PRL 87 (2001) 168501.

[15] R. Peierls, Ann. Phys. 3 (1029) 1055.

[16] R. Brout, I. Prigogine, Physica 22 (1956) 621-636.

[17] G.M. Zaslavskii, R.Z. Sagdeev, Sov. Phys. JETP 25 (1967) 718.

[18] V.E. Zakharov, P. Guyenne, A.N. Pushkarev, F. Dias, Physica D 152-153 (2001) 573-619. 
[19] Yu. Lvov, Sergey Nazarenko, Spectral fluctuations, long correlations and intermittency in wave turbulence, Phys. Rev. E 69 (2004) 066608.

[20] Y. Choi, Yu. Lvov, S.V. Nazarenko, Anomalous probability of high amplitudes in wave turbulence, PLA (submitted for publication). Also at http://www.arxiv.org/abs/math-ph/0404022.

[21] L. Biven, S.V. Nazarenko, A.C. Newell, Breakdown of wave turbulence and the onset of intermittency, Phys. Lett. A 280 (2001) 28-32; A.C. Newell, S.V. Nazarenko, L. Biven, Physica D 152-153 (2001) 520-550.

[22] P.A.E.M. Janssen, Nonlinear four-wave interactions and freak waves, J. Phys. Oceanography 33 (2003) 864. 